University of Texas Rio Grande Valley

ScholarWorks @ UTRGV

Physics and Astronomy Faculty Publications and Presentations

6-1-2018

\title{
The Green Bank North Celestial Cap Pulsar Survey. III. 45 New Pulsar Timing Solutions
}

Ryan S. Lynch

Joseph K. Swiggum

Vlad I. Kondratiev

David L. Kaplan

Kevin Stovall

See next page for additional authors

Follow this and additional works at: https://scholarworks.utrgv.edu/pa_fac

Part of the Astrophysics and Astronomy Commons

\section{Recommended Citation}

Ryan S. Lynch, et. al., (2018) The Green Bank North Celestial Cap Pulsar Survey. III. 45 New Pulsar Timing Solutions.Astrophysical Journal859:2. DOI: http://doi.org/10.3847/1538-4357/aabf8a

This Article is brought to you for free and open access by the College of Sciences at ScholarWorks @ UTRGV. It has been accepted for inclusion in Physics and Astronomy Faculty Publications and Presentations by an authorized administrator of ScholarWorks @ UTRGV. For more information, please contact justin.white@utrgv.edu, william.flores01@utrgv.edu. 


\section{Authors}

Ryan S. Lynch, Joseph K. Swiggum, Vlad I. Kondratiev, David L. Kaplan, Kevin Stovall, Emmanuel Fonseca, Mallory S.E. Roberts, Lina Levin, Megan E. Decesar, Bingyi Cui, S. Bradley Cenko, Pradip Gatkine, Anne M. Archibald, Shawn Banaszak, Christopher M. Biwer, Jason Boyles, Pragya Chawla, Louis P. Dartez, David Day, Anthony J. Ford, Joseph Flanigan, Jason W.T. Hessels, Jesus Hinojosa, Fredrick A. Jenet, Chen Karako-Argaman, Victoria M. Kaspi, Sean Leake, Grady Lunsford, José G. Martinez, and Alberto Mata 


\title{
The Green Bank North Celestial Cap Pulsar Survey. III. 45 New Pulsar Timing Solutions
}

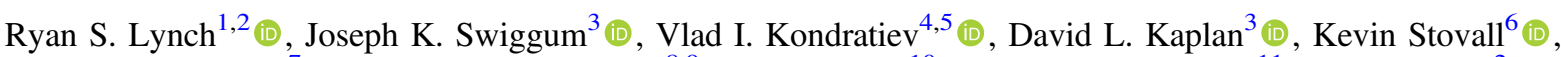 \\ Emmanuel Fonseca $^{7}$ (1), Mallory S. E. Roberts ${ }^{8,9}$ (1) Lina Levin $^{10}$ (i), Megan E. DeCesar ${ }^{11}$ (i), Bingyi Cui ${ }^{2}$ (i),
} S. Bradley Cenko ${ }^{12,13}$ (D) , Pradip Gatkine ${ }^{14}$ (D) , Anne M. Archibald ${ }^{4}$ (iD), Shawn Banaszak ${ }^{3}$, Christopher M. Biwer ${ }^{15}$, Jason Boyles ${ }^{16}$, Pragya Chawla ${ }^{7}$ (1), Louis P. Dartez ${ }^{17}$, David Day ${ }^{17}$, Anthony J. Ford ${ }^{17}$, Joseph Flanigan ${ }^{3}$, Jason W. T. Hessels ${ }^{4,18}$ (1) Jesus Hinojosa $^{17}$, Fredrick A. Jenet ${ }^{17}$, Chen Karako-Argaman ${ }^{7}$, Victoria M. Kaspi ${ }^{7}$ (i), Sean Leake ${ }^{17}$, Grady Lunsford ${ }^{17}$, José G. Martinez $^{19}$, Alberto Mata ${ }^{17}$, Maura A. McLaughlin ${ }^{2}$ (1), Hind Al Noori ${ }^{8}$ (1), Scott M. Ransom ${ }^{20}{ }^{(1)}$, Matthew D. Rohr $^{3}$, Xavier Siemens $^{3}$, Renée Spiewak ${ }^{3,21}$ (1) , Ingrid H. Stairs ${ }^{22}$ (1) , Joeri van Leeuwen ${ }^{4,18}$ (1), Arielle N. Walker ${ }^{3}$, and Bradley L. Wells ${ }^{3,23}$

${ }^{1}$ Green Bank Observatory, P.O. Box 2, Green Bank, WV 24494, USA; rlynch@nrao.edu

${ }^{2}$ Center for Gravitational Waves and Cosmology, Department of Physics and Astronomy, West Virginia University, White Hall, Box 6315, Morgantown, WV 26506, USA

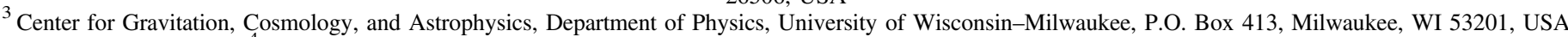
${ }_{5}^{4}$ ASTRON, the Netherlands Institute for Radio Astronomy, Postbus 2, 7990 AA, Dwingeloo, The Netherlands

${ }^{5}$ Astro Space Center of the Lebedev Physical Institute, Profsoyuznaya str. 84/32, Moscow, 117997, Russia

${ }^{6}$ National Radio Astronomy Observatory, 1003 Lopezville Rd., Socorro, NM 87801, USA

${ }^{7}$ Department of Physics \& McGill Space Institute, McGill University, 3600 University Street, Montreal, QC H3A 2T8, Canada ${ }^{8}$ New York University Abu Dhabi, Abu Dhabi, UAE

${ }^{9}$ Eureka Scientific, Inc., 2452 Delmer St., Suite 100, Oakland, CA 94602, USA

${ }^{10}$ Jodrell Bank Centre for Astrophysics, School of Physics and Astronomy, The University of Manchester, Manchester, M13 9PL, UK

${ }^{11}$ Department of Physics, 730 High St., Lafayette College, Easton, PA 18042, USA

12 Astrophysics Science Division, NASA Goddard Space Flight Center, Mail Code 661, Greenbelt, MD 20771, USA

13 Joint Space-Science Institute, University of Maryland, College Park, MD 20742, USA

${ }^{14}$ Department of Astronomy, University of Maryland, College Park, MD 20742, USA

${ }^{15}$ Department of Physics, Syracuse University, Syracuse, NY 13244, USA

${ }^{16}$ Department of Physics and Astronomy, Western Kentucky University, 1906 College Heights Blvd., Bowling Green, KY 42101, USA

${ }^{17}$ Center for Advanced Radio Astronomy, University of Texas Rio Grande Valley, 1 W. University Blvd., Brownsville, TX 78520, USA

18 Anton Pannekoek Institute for Astronomy, University of Amsterdam, Science Park 904, 1098 XH, Amsterdam, The Netherlands

${ }^{19}$ Max-Planck-Institut fü Radioastronomie, Auf dem Hügel 69, D-53121, Bonn, Germany

${ }^{20}$ National Radio Astronomy Observatory, 520 Edgemont Road, Charlottesville, VA 23903, USA

${ }^{21}$ Centre for Astrophysics and Supercomputing, Swinburne University of Technology, P.O. Box 218, Hawthorn, VIC 3122, Australia

${ }^{22}$ Department of Physics and Astronomy, University of British Columbia, 6224 Agriculture Rd., Vancouver, BC V6T 1Z1, Canada

${ }^{23}$ Department of Atmospheric Sciences, Colorado State University, 3915 W. Laporte Ave., Fort Collins, CO 80523, USA Received 2018 January 7; revised 2018 April 16; accepted 2018 April 17; published 2018 May 29

\begin{abstract}
We provide timing solutions for 45 radio pulsars discovered by the Robert C. Byrd Green Bank Telescope. These pulsars were found in the Green Bank North Celestial Cap pulsar survey, an all-GBT-sky survey being carried out at a frequency of $350 \mathrm{MHz}$. We include pulsar timing data from the Green Bank Telescope and Low Frequency Array. Our sample includes five fully recycled millisecond pulsars (MSPs, three of which are in a binary system), a new relativistic double neutron star system, an intermediate-mass binary pulsar, a mode-changing pulsar, a $138 \mathrm{~ms}$ pulsar with a very low magnetic field, and several nulling pulsars. We have measured two post-Keplerian parameters and thus the masses of both objects in the double neutron star system. We also report a tentative companion mass measurement via Shapiro delay in a binary MSP. Two of the MSPs can be timed with high precision and have been included in pulsar timing arrays being used to search for low-frequency gravitational waves, while a third MSP is a member of the black widow class of binaries. Proper motion is measurable in five pulsars, and we provide an estimate of their space velocity. We report on an optical counterpart to a new black widow system and provide constraints on the optical counterparts to other binary MSPs. We also present a preliminary analysis of nulling pulsars in our sample. These results demonstrate the scientific return of long timing campaigns on pulsars of all types.
\end{abstract}

Key words: proper motions - pulsars: general - surveys

Supporting material: extended figure

\section{Introduction}

Radio pulsars have long been used as exquisite natural laboratories for studying a wide range of phenomena in physics and astronomy. The well-known double neutron star (DNS) system PSR B1913+16 provided the first observational evidence for the existence of gravitational waves (GWs; Taylor \& Weisberg 1989; Weisberg et al. 2010), and the double pulsar system J0737-3039 continues to place ever more stringent constraints on deviations from general relativity (GR) in the strong-field regime (Kramer et al. 2006). Neutron star mass measurements can be used to study nuclear physics and the equation of state of ultradense matter (Demorest et al. 2010) while also providing insight into the mass distribution of the neutron star population (Özel et al. 2012; Kiziltan et al. 2013; Antoniadis et al. 2016) and, in turn, formation mechanisms and evolution (e.g., Lattimer \& Prakash 2004; Tauris \& Savonije 1999). Proper-motion measurements can be used to estimate transverse velocity, which also informs theories of 
neutron star formation and supernova energetics (Ferdman et al. 2013). While most binary pulsars have low-mass He white dwarf (WD) companions, rarer binaries have also been found. These include systems with more massive CO WDs (e.g., Camilo et al. 2001), giant companions that are actively transferring mass to the neutron star, and so-called black widow binaries, where an energetic pulsar wind has ablated the companion's outer layers, leaving a very low-mass degenerate core (Roberts 2013).

High-precision millisecond pulsars (MSPs, which we define as $P<10 \mathrm{~ms}$ ) are currently being used in an effort to directly detect nanohertz-frequency GWs by forming a pulsar timing array (PTA). These GWs are predicted to cause nanosecondscale deviations in pulse arrival times with a unique angular correlation between pairs of MSPs. At nanohertz frequencies, the dominant source class is expected to be supermassive binary black holes in the early stages of inspiral, though more exotic sources such as cosmic strings are also predicted to emit at these $\mathrm{GW}$ frequencies. One of the best ways to improve the sensitivity of PTAs is by adding new high-precision pulsars to the array, particularly on angular baselines that are not currently well sampled (Siemens et al. 2013). PTAs are a major project at all large radio telescopes: the North American Nanohertz Observatory for Gravitational Waves (NANOGrav; McLaughlin 2013) uses the Robert C. Byrd Green Bank Telescope (GBT) and William E. Gordon telescope at the Arecibo Observatory, the Parkes Pulsar Timing Array (PPTA; Hobbs 2013) uses the Parkes Telescope, and the European Pulsar Timing Array (EPTA; Kramer \& Champion 2013) uses the Effelsberg Telescope, Lovell Telescope, Nançay Radio Telescope, Sardinia Radio Telescope, and Westerbork Synthesis Array. All of these projects also collaborate under the framework of the International Pulsar Timing Array (Manchester \& IPTA 2013).

Despite many decades of investigation, pulsar emission mechanisms are not fully understood. A wide variety of behavior is observed, however. This includes abrupt changes in the average pulse profile between a small number of modes that may be accompanied by changes in the spin-down rate of the pulsar and are tied to global magnetospheric reconfigurations (Lyne et al. 2010). Pulsar emission may also be variable on a variety of timescales: so-called nulling pulsars may cease radio emission in as short as one rotation, remain in a quiescent state for many rotations, and then switch back on just as suddenly. First discovered by Backer (1970), nulling pulsars are an invaluable population for studying pulsar emission mechanisms and magnetospheres. Despite nearly 50 years of investigation, nulling remains poorly understood, and only $\sim 130$ pulsars ( $\sim 5 \%$ of the known population; Gajjar 2017) exhibit nulling behavior. In the extreme case of rotating radio transients (RRATs; McLaughlin et al. 2006), only a few single pulses are ever observed.

It is essential to conduct long-term timing campaigns on new pulsars if their scientific impact is to be fully realized. The arrival times of a fiducial point in a pulsar's light curve are measured to high precision and used as input to a model that is coherent in rotational phase. Deviations from the predicted arrival times reveal information about the pulsar and its environment, such as the rotational period and period derivative, astrometric parameters, column density of electrons along the line of sight, and Keplerian and post-Keplerian orbital parameters. Because pulsar timing accounts for every rotation of the pulsar, parameters can be measured with remarkable precision. The observed rotational parameters can be used to derive canonical properties such as characteristic age, surface magnetic field strength, and total spin-down luminosity. Long-term timing campaigns can be used to measure even small effects with high significance, and regular monitoring makes it possible to study time-variable phenomena such as mode-changing and nulling.

Large-area surveys are the best way to find new and interesting pulsars. The Green Bank North Celestial Cap (GBNCC) pulsar survey is an ongoing $350 \mathrm{MHz}$ all-sky search for pulsars and transients being carried out with the GBT (Stovall et al. 2014). With 160 pulsars and RRATs discovered to date, it is the most successful pulsar survey conducted in this frequency range.

In this paper, we report pulsar timing solutions for 45 pulsars discovered by the GBNCC survey. These include five MSPs (two of which are being timed by NANOGrav and the IPTA); five binary pulsars, including a relativistic DNS system; an intermediate-mass binary pulsar (IMBP); a black widow system; a long-period pulsar with an anomalously low magnetic field; a mode-changing pulsar; and six nulling pulsars. We have measured the masses of both constituents of the DNS system and also report a tentative mass measurement via Shapiro delay of a WD companion to an MSP. An additional 10 pulsars, including one MSP, one disrupted recycled pulsar, and one nulling pulsar are reported on in Kawash et al. (2018). In Section 2, we provide a brief overview of the GBNCC survey, and in Section 3, we describe the observational setups that were used in our timing campaign. In Section 4, we provide details of the data reduction and timing analysis. In Section 5, we provide timing solutions and discuss select individual systems; in Section 6, we present our nulling analysis; and in Section 7, we discuss constraints on optical companions to the binary pulsars. We summarize our results in Section 8.

\section{The GBNCC Pulsar Survey}

Stovall et al. (2014) described the GBNCC survey in detail; here we provide a brief overview. The survey began in 2009 covering declinations $\delta>38^{\circ}$ and has continued to cover the full sky visible from the GBT ( $85 \%$ of the celestial sphere). It is being carried out at a center frequency of $350 \mathrm{MHz}$ and with dwell times of $120 \mathrm{~s}$. The low observing frequency preferentially selects sources with low dispersion measures (DMs; the electron column density) and steep spectral indices relative to surveys at higher frequencies. Total intensity data are collected using the Green Bank Ultimate Pulsar Processing Instrument (GUPPI) using a bandwidth of $100 \mathrm{MHz}, 4096$ frequency channels, and $81.92 \mu$ s sampling time. Data are processed on the Guillimin high-performance computer operated by McGill University, Compute Canada, and Calcul Québec using a pipeline based on the PRESTO ${ }^{24}$ software package (Ransom et al. 2002) and are searched for periodic signals and single pulses. Candidates are uploaded to a web-based image-viewing and ranking application. ${ }^{25}$ Periodicity candidates are analyzed with a pattern-recognition neural net (Zhu et al. 2014), and single-pulse candidates are analyzed with a grouping algorithm (Karako-Argaman et al. 2015; Chawla et al. 2017). Recently, a

\footnotetext{
24 http://www.cv.nrao.edu/ sransom/presto

25 Hosted on http://ca.cyberska.org/.
} 
Table 1

Observing Setup

\begin{tabular}{|c|c|c|c|c|c|c|}
\hline \multirow{2}{*}{ Receiver } & \multirow{2}{*}{$f_{\text {ctr }}(\mathrm{MHz})$} & \multirow{2}{*}{$\Delta f(\mathrm{MHz})$} & \multicolumn{2}{|c|}{$\begin{array}{c}\text { Incoherent } \\
\text { Dedispersion }\end{array}$} & \multicolumn{2}{|c|}{$\begin{array}{c}\text { Coherent } \\
\text { Dedispersion }\end{array}$} \\
\hline & & & $n_{\text {chan }}$ & $t_{\text {int }}(\mu \mathrm{s})$ & $n_{\text {chan }}$ & $t_{\text {int }}(\mu \mathrm{s})$ \\
\hline LOFAR & 148 & 78 & 6400 & 327.68 & 400 & 5.12 \\
\hline PF 342 & 350 & 100 & 4096 & 81.92 & 512 & 10.24 \\
\hline PF 800 & 820 & 200 & 2048 & 40.96 & 512 & 10.24 \\
\hline $\mathrm{L}$ band & 1500 & 800 & 2048 & 40.96 & 512 & 10.24 \\
\hline $\mathrm{S}$ band & 2000 & 800 & 2048 & 40.96 & $\cdots$ & $\cdots$ \\
\hline
\end{tabular}

fast-folding algorithm for periodicity candidates (Parent et al. in preparation) and a neural-net classifier for single-pulse candidates have also been implemented.

To date, $\sim 75 \%$ of the full survey area has been covered. A total of 156 pulsars have been discovered, ${ }^{26}$ including 20 MSPs and 11 RRATs (Stovall et al. 2014; Karako-Argaman et al. 2015). Data collection is projected to finish in 2020 .

\section{Observational Setup and Data Collection}

We used the GBT and the Low Frequency Array (LOFAR) for initial follow-up and timing. The observational parameters for each instrument are presented below and summarized in Table 1.

\subsection{GBT Observations}

We collected all GBT data with GUPPI in the PSRFITS format. Dedicated timing observations began in 2013 January, though data from confirmation observations and test observations during the primary survey have also been included when available. All pulsars were observed regularly for a minimum of one full year, and observations have continued for select MSPs and binary pulsars. We used a variety of observing frequencies and instrumental setups, but most data were collected using the GBT's prime focus receiver at a center frequency of $820 \mathrm{MHz}$. The pulsars' initial positions were only known to a precision of $\sim 36^{\prime}$ (the half-power beamwidth of the GBT at $350 \mathrm{MHz}$ ), so we first obtained a refined position using a seven-point grid map at $820 \mathrm{MHz}$ (Morris et al. 2002). During preliminary observations, we used GUPPI in its incoherent dedispersion mode, but as timing solutions improved, we also used a coherent dedispersion mode. When observing with incoherent dedispersion, we recorded total intensity filter-bank data. In coherent dedispersion modes, we recorded all four Stokes parameters and folded the data in realtime modulo the instantaneous pulsar period, recording subintegrations every $10 \mathrm{~s}$. Observing times varied between sources and sessions but were typically 5-15 minutes.

\subsection{LOFAR Observations}

A subset of pulsars discovered in the GBNCC survey, including those presented in this paper, were also initially followed up and timed with LOFAR in the frequency range of 110-188 MHz. Observations were carried out for almost 2 years, from 2013 March 6 until 2015 January 14, during LOFAR's Cycles 0-2 (project codes LC0_022, LC1_025, and LC2_007). For every pulsar, one or several gridding observations were first

\footnotetext{
${ }^{26}$ For an up-to-date list, see http://astro.phys.wvu.edu/GBNCC/.
}

performed to improve the accuracy of the discovery position to within $2^{\prime}-3^{\prime}$ by forming coherent tied-array beams (TABs) around the nominal position (e.g., Karako-Argaman et al. 2015). In some cases, the first timing observations were also carried out with an extra one to two rings of TABs ( 7 or 19 beams, ring size of $2 ! 5)$ to further refine the position. All observations were conducted with the LOFAR's Full Core using 42-48 HBA substations in most observations but not less than 38 substations. For the timing solutions presented in this paper, only LOFAR timing (not gridding) observations are used. Timing observations were performed with roughly a monthly cadence. Most observations were 5 minutes long, except for the mode-changing pulsar $\mathrm{J} 1628+4406$. which was observed for 60 minutes at a time to increase the chances of catching a transition between the modes. We include LOFAR data for 29 of the pulsars presented here.

For the initial gridding and timing observations of slow pulsars and RRATs, we recorded Stokes $I$ data in a $78.125 \mathrm{MHz}$ band centered at about $149 \mathrm{MHz}$ split into 400 subbands (subband numbers 51-450). Each subband in turn was split into 16 channels sampled at $327.68 \mu$ s. This setup was also used for timing observations of PSR J1628+4406. All subsequent timing observations of slow pulsars and MSPs (during the LC1_035 and LC2_007 projects) were carried out recording 400 subbands of complex-voltage $(\mathrm{CV})$ data sampled at $5.12 \mu \mathrm{s}$ in the same frequency range. The LOFAR PULsar Pipeline (PULP) was run after the observation to dedisperse (coherently for the CV mode) and clean the data and fold it using the best available pulsar ephemeris. The length of the subintegrations was $5 \mathrm{~s}$ for MSPs and $60 \mathrm{~s}$ for slow pulsars. The folded PSRFITS archive files, together with other pipeline data products including different diagnostic plots, were ingested to the LOFAR's Long Term Archive. For a more detailed description of the observing setup and PULP, see Kondratiev et al. (2016).

\section{Pulsar Timing Analysis}

Since the rotational parameters of the pulsars were not initially known to high precision, we processed early data using PRESTO to excise radio frequency interference (RFI), search for the pulsars at each epoch, and fold the data at optimal periods, period derivatives, and DMs. When pulsars are in a binary system, orbital acceleration causes a Doppler modulation of the observed rotational period that is sinusoidal in the case of nearly circular orbits but that can take on a more complicated form when the orbital eccentricity is high. Binary pulsars were observed at high cadence to sample a range of orbital phases, and we used a least-squares optimizer to fit a sinusoid to the observed rotational periods (and period derivatives, when measurable). This in turn yielded a lowprecision estimate of the Keplerian orbital parameters, which were used as a starting point in our timing solutions. PSR J0509+3801 was soon found to have a high eccentricity (see Section 5.3.1). Initial orbital parameters were found by following Bhattacharyya \& Nityananda (2008) in combination with a least-squares optimizer that fits nonsinusoidal period modulations.

We calculated pulse times of arrival (TOAs) via Fourierdomain cross-correlation of a noise-free template and the observed pulse profile (Taylor 1992). Initial templates were created by fitting one or more Gaussians to the pulse profiles. Final templates were made by phase-coherently adding all high 
signal-to-noise $(\mathrm{S} / \mathrm{N})$ data at a given frequency and then denoising the summed profile via wavelet smoothing. When there was minimal evolution of the pulse profile between different observing bands, we were able to align templates at different frequencies. When this was not possible, we allowed for an arbitrary offset in our timing models between TOAs from different bands. At each epoch, we summed the data in time and frequency to create $60 \mathrm{~s}$ subintegrations and four frequency subbands and calculated a TOA for each. In some cases, visual inspection of the resulting residuals revealed a large number of outliers. To increase the $\mathrm{S} / \mathrm{N}$, we further summed the data to obtain single-frequency TOAs or a single TOA per observing session. A small number of individual TOAs still had very large residuals and were found to suffer from especially low $\mathrm{S} / \mathrm{N}$ or, more often, were contaminated by RFI. These TOAs were removed from further analysis.

We used the TEMPO ${ }^{27}$ pulsar timing program to fit a timing model to the observed TOAs. The basic model consists of rotational frequency and frequency derivative, position, and DM. In some cases, we were also able to measure a significant proper motion. When appropriate, we also fit for Keplerian and post-Keplerian orbital parameters. All final timing solutions were derived using ecliptic coordinates $(\lambda, \beta)$, which are nearly orthogonal in timing models and thus provide a more accurate representation of the error ellipse (Matthews et al. 2016). We use the DE430 planetary ephemeris and TT(BIPM) time standard as implemented in TEMPO. Most of the binary systems presented here have very low eccentricity, so we use the ELL1 timing model (Lange et al. 2001), which parameterizes the orbit in terms of the epoch of the ascending node and the first and second Laplace-Lagrange parameters,

$$
\begin{aligned}
T_{\mathrm{asc}} & =T_{0}-P_{\mathrm{b}} \frac{\omega}{2 \pi}, \\
\epsilon_{1} & =e \sin \omega, \\
\epsilon_{2} & =e \cos \omega,
\end{aligned}
$$

where $T_{0}$ is the epoch of periastron passage, $P_{\mathrm{b}}$ is the binary period, $\omega$ is the longitude of periastron passage, and $e$ is the eccentricity. This parameterization is appropriate for systems where $e^{2} a / c \sin i$ is much less than the errors in the TOA measurements (here $a$ is the semimajor axis, $i$ is the inclination angle, and $c$ is the speed of light). The exception is PSR J0509 +3801 , a highly eccentric DNS system.

\section{Results}

The measured rotational parameters and common derived properties of all of our pulsars are shown in Tables 2 and 3, respectively. Table 4 shows positions in a variety of coordinate systems, as well as DMs. The ELL1 binary timing parameters of four pulsars are shown in Table 5. The locations of all of the pulsars presented here on the $P-\dot{P}$ diagram are shown in Figure 1. Post-fit timing residuals and integrated pulse profiles are shown in Figures 8 and 9, respectively. In the following sections, we present specific details on pulsars of interest.

\subsection{NANOGrav and IPTA Pulsars}

One of the primary science goals of the GBNCC survey is finding high-precision MSPs for inclusion in PTAs. The quadrupolar nature of GWs should cause a unique angular

\footnotetext{
${ }^{27}$ http://tempo.sourceforge.net/
}

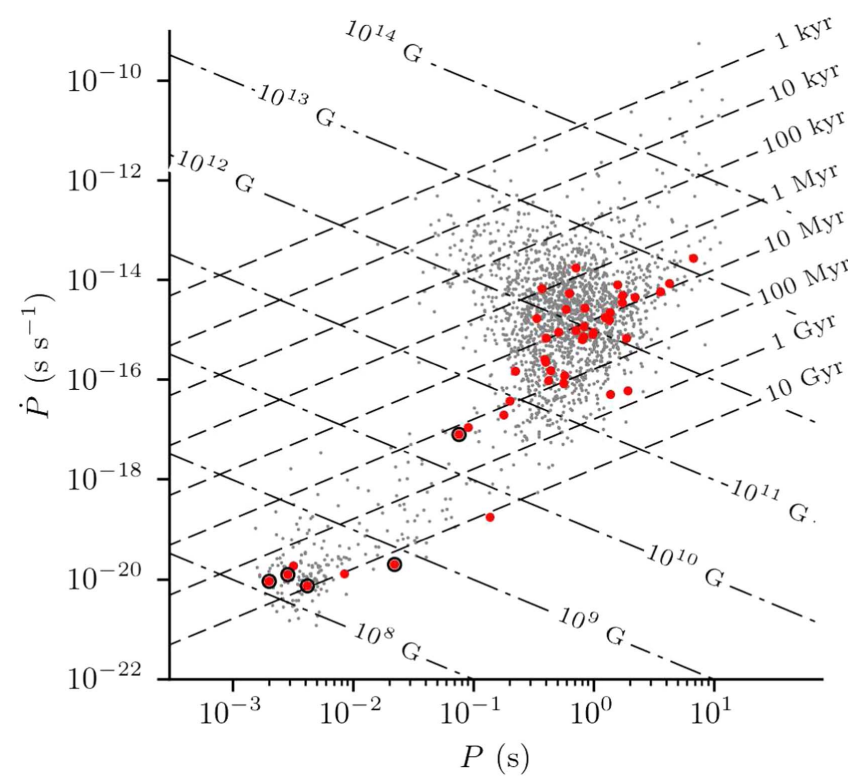

Figure 1. Spin period vs. period derivative for the 45 pulsars presented here (red circles), as well as all of the pulsars listed in the ATNF pulsar catalog (http://www.atnf.csiro.au/research/pulsar/psrcat) (gray points; Manchester et al. 2005). Binary pulsars are indicated with a black circle. Dashed lines show constant characteristic age, and dot-dashed lines show constant surface magnetic field.

correlation between pairs of MSPs in a PTA. Sampling many baselines is essential to firmly establish this quadrupolar signature, and this will be necessary for PTAs to claim a detection of low-frequency GWs. The GBNCC survey is especially well suited to find MSPs at high northern declinations, which have been historically undersampled by sensitive pulsar surveys.

Two MSPs presented here have been included in NANOGrav and the IPTA based on promising early results: PSRs $\mathrm{J} 0740+6620$ and $\mathrm{J} 1125+7819$. We found it necessary to use the DMX timing model for PSR J1125+7819 to measure epoch-dependent variations in DM. We used a 30 day window for DMX epochs and found typical DM variations of $\sim 10^{-4}-10^{-5} \mathrm{pc} \mathrm{cm}^{-3}$, with a maximum $|\Delta \mathrm{DM}|=1.7 \times$ $10^{-2} \mathrm{pc} \mathrm{cm}^{-3}$. These results are consistent with DM variations measured in NANOGrav timing ${ }^{28}$ (Arzoumanian et al. 2018). PSRs J1710+4923 and J1641+8049 have not been included in PTAs despite a relatively low uncertainty on individual TOAs -PSR J1710+4923 because of strong scintillation and PSR $\mathrm{J} 1641+8049$ because it is in a black widow binary system.

\subsection{Proper Motions and Kinematic Corrections}

We have measured timing proper motions for five MSPs: PSRs J0740+6620, J1125+7819, J1641+8049, J1710+4923, and J1955+6708. Table 6 lists the measured proper motions, estimated transverse velocity, $v_{\mathrm{t}}$ calculated using the DMinferred distances in both the NE2001 Cordes \& Lazio (2002) and YMW16 (Yao et al. 2017) models, and kinematic corrections.

\footnotetext{
$\overline{28}$ NANOGrav observes PSR J1125+7819 at 820 and $1500 \mathrm{MHz}$ using coherent dedispersion, with 30 minute integrations per TOA. Nearly all of our timing data were taken at 350 and $820 \mathrm{MHz}$ but with incoherent dedispersion and shorter integration times. As such, NANOGrav achieves better rms timing residuals.
} 
Table 2

Rotational and Timing Parameters of GBNCC Pulsars

\begin{tabular}{|c|c|c|c|c|c|c|c|c|}
\hline PSR & $\begin{array}{c}\text { Name Used in } \\
\text { Stovall et al. (2014) }\end{array}$ & $\begin{array}{c}\nu \\
(\mathrm{Hz})\end{array}$ & $\begin{array}{c}\dot{\nu} \\
\left(\mathrm{Hz} \mathrm{s}^{-1}\right)\end{array}$ & $\begin{array}{l}\text { Epoch } \\
\text { (MJD) }\end{array}$ & $\begin{array}{l}\text { Data Span } \\
\text { (MJD) }\end{array}$ & $\begin{array}{c}\text { rms Residual } \\
(\mu \mathrm{s})\end{array}$ & $N_{\text {TOA }}$ & $\chi_{\mathrm{red}}^{2}$ \\
\hline J0054+6946 & $\mathrm{J} 0053+69$ & $1.200607994501(6)$ & $-1.037(1) \times 10^{-15}$ & 56500.0 & $56329-56791$ & 542.59 & 297 & $\overline{0.9}$ \\
\hline $\mathrm{J} 0058+4950$ & $\mathrm{~J} 0059+50$ & $1.00399242379(4)$ & $-8.1(2) \times 10^{-16}$ & 56500.0 & $56358-56670$ & 1701.9 & 186 & 1.9 \\
\hline $\mathrm{J} 0137+6349$ & $\mathrm{~J} 0136+63$ & $1.39282996541(1)$ & $-1.862(9) \times 10^{-15}$ & 56500.0 & $56329-56670$ & 797.51 & 276 & 1.3 \\
\hline $\mathrm{J} 0212+5222$ & $\mathrm{~J} 0213+52$ & $2.65684439046(1)$ & $-4.6583(9) \times 10^{-14}$ & 56500.0 & $56332-56670$ & 376.92 & 252 & 1.6 \\
\hline $\mathrm{J} 0325+6744$ & $\mathrm{~J} 0325+67$ & $0.732773180015(8)$ & $-8.34(5) \times 10^{-16}$ & 56500.0 & $56351-56669$ & 952.49 & 204 & 2.6 \\
\hline $\mathrm{J} 0358+6627$ & $\mathrm{~J} 0358+66$ & $10.92827132616(2)$ & $-1.30(1) \times 10^{-15}$ & 56500.0 & $56329-56669$ & 80.73 & 86 & 0.9 \\
\hline $\mathrm{J} 0509+3801$ & $\mathrm{~J} 0510+38$ & $13.06483380156(2)$ & $-1.3538(4) \times 10^{-15}$ & 56900.0 & $56336-57474$ & 102.36 & 791 & 1.1 \\
\hline $\mathrm{J} 0518+5416$ & $\mathrm{~J} 0519+54$ & $2.93942447332(5)$ & $-1.448(3) \times 10^{-14}$ & 56500.0 & $56336-56669$ & 961.97 & 207 & 3. \\
\hline $\mathrm{J} 0612+3721$ & $\mathrm{~J} 0610+37$ & $2.2529049469(1)$ & $-7.6(7) \times 10^{-16}$ & 56500.0 & $56336-56669$ & 1373.35 & 69 & 1.0 \\
\hline $\mathrm{J} 0738+6904$ & $\mathrm{~J} 0737+69$ & $0.14646235983(1)$ & $-5.785(8) \times 10^{-16}$ & 56425.0 & $56159-56669$ & 2138.93 & 84 & 4. \\
\hline $\mathrm{J} 0740+6620$ & $\mathrm{~J} 0741+66$ & $346.53199660394(1)$ & $-1.4658(8) \times 10^{-15}$ & 56675.0 & $56156-56908$ & 2.95 & 383 & 1 \\
\hline $\mathrm{J} 1624+8643$ & $\mathrm{~J} 1627+86$ & $2.52676461497(1)$ & $-1.625(6) \times 10^{-15}$ & 56500.0 & $56351-56669$ & 223.5 & 279 & 1.1 \\
\hline $\mathrm{J} 1628+4406$ & $\mathrm{~J} 1629+43$ & $5.519419001073(2)$ & $-5.9032(8) \times 10^{-16}$ & 56625.0 & $56017-56973$ & 99.14 & 2225 & 3.7 \\
\hline $\mathrm{J} 1641+8049$ & $\mathrm{~J} 1649+80$ & $494.76063707093(2)$ & $-2.19(2) \times 10^{-15}$ & 56425.0 & $56159-56670$ & 5.81 & 788 & 2.7 \\
\hline $\mathrm{J} 1647+6608$ & $\mathrm{~J} 1647+66$ & $0.62507876955(1)$ & $-3.063(6) \times 10^{-15}$ & 56500.0 & $56351-56669$ & 690.08 & 174 & 1.2 \\
\hline $\mathrm{J} 1710+4923$ & $\mathrm{~J} 1710+49$ & $310.536979440897(4)$ & $-1.7561(2) \times 10^{-15}$ & 56850.0 & $55996-57700$ & 10.76 & 757 & 2. \\
\hline $\mathrm{J} 1800+5034$ & $\mathrm{~J} 1800+50$ & $1.72898175945(3)$ & $-3.5(2) \times 10^{-16}$ & 56500.0 & $56365-56670$ & 814.31 & 338 & 1 \\
\hline $\mathrm{J} 1815+5546$ & $\mathrm{~J} 1815+55$ & $2.3427772328(2)$ & $-5.2(8) \times 10^{-16}$ & 56500.0 & $56365-56670$ & 1800.71 & 103 & 4 \\
\hline $\mathrm{J} 1821+4147$ & $\mathrm{~J} 1821+41$ & $0.792482693574(5)$ & $-1.0860(6) \times 10^{-15}$ & 56375.0 & $56085-56670$ & 625.93 & 252 & \\
\hline $\mathrm{J} 1859+7654$ & $\mathrm{~J} 1859+76$ & $0.71749953039(1)$ & $-2.6(8) \times 10^{-17}$ & 56500.0 & $56351-56669$ & 682.37 & 185 & 1 \\
\hline $\mathrm{J} 1923+4243$ & $\mathrm{~J} 1921+42$ & $1.68012768584(3)$ & $-7.15(1) \times 10^{-15}$ & 56525.0 & $56365-56670$ & 520.97 & 228 & 1.1 \\
\hline $\mathrm{J} 1934+5219$ & $\mathrm{~J} 1935+52$ & $1.75919355395(8)$ & $-2.6(4) \times 10^{-16}$ & 56525.0 & $56365-56670$ & 2086.41 & 178 & 2.3 \\
\hline $\mathrm{J} 1938+6604$ & $\mathrm{~J} 1939+66$ & $44.926049244297(5)$ & $-3.93(7) \times 10^{-17}$ & 56550.0 & $56176-56907$ & 28.02 & 691 & 1.4 \\
\hline $\mathrm{J} 1941+4320$ & $\mathrm{~J} 1941+43$ & $1.189192897888(8)$ & $-1.6224(7) \times 10^{-15}$ & 56375.0 & $56081-56670$ & 848.81 & 286 & 1.9 \\
\hline $\mathrm{J} 2208+4056$ & $\mathrm{~J} 2207+40$ & $1.56996372132(3)$ & $-1.3022(2) \times 10^{-14}$ & 56375.0 & $56081-56670$ & 1882.71 & 235 & 1.9 \\
\hline $\mathrm{J} 2228+6447$ & $\mathrm{~J} 2229+64$ & $0.52826709593(1)$ & $-1.87(8) \times 10^{-16}$ & 56500.0 & $56329-56670$ & 1868.72 & 209 & 1.1 \\
\hline $\mathrm{J} 2241+6941$ & $\mathrm{~J} 2243+69$ & $1.16904199244(2)$ & $-3.68(1) \times 10^{-15}$ & 56500.0 & $56351-56670$ & 889.58 & 173 & 1.4 \\
\hline $\mathrm{J} 2310+6706$ & $\ldots$ & $0.5141946062(3)$ & $-2(1) \times 10^{-17}$ & 57225.0 & $57078-57397$ & 1204.93 & 80 & 2. \\
\hline $\mathrm{J} 2312+6931$ & $\mathrm{~J} 2316+69$ & $1.22944554793(3)$ & $-9.5(2) \times 10^{-16}$ & 56500.0 & $56331-56670$ & 1657.42 & 146 & 2.4 \\
\hline $\mathrm{J} 2351+8533$ & $\mathrm{~J} 2353+85$ & $0.98840874179(6)$ & $-8.6(3) \times 10^{-16}$ & 56500.0 & $56332-56669$ & 1398.72 & 42 & 1.1 \\
\hline
\end{tabular}

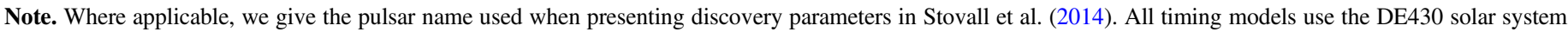
ephemeris and are referenced to the TT(BIPM) time standard. Values in parentheses are the $1 \sigma$ uncertainty in the last digit as reported by TEMPO.

The observed pulsar spin down is a contribution of several effects,

$$
\dot{P}_{\mathrm{obs}}=\dot{P}_{\mathrm{int}}+\dot{P}_{\mathrm{G}}+\dot{P}_{\mathrm{S}}
$$

where $\dot{P}_{\mathrm{G}}$ is caused by acceleration between the pulsar and solar system barycenter in a differential Galactic potential and $\dot{P}_{\mathrm{s}}$ is the Shklovskii effect for pulsars. Following Nice \& Taylor (1995), the bias arising from acceleration perpendicular to the Galactic plane $\left(a_{z}\right)$ is given by

$$
\frac{\dot{P}_{\mathrm{G}, \perp}}{P}=\frac{a_{z} \sin b}{c}
$$

where $b$ is the Galactic latitude and $a_{z}$ is

$$
\frac{a_{z}}{c}=-1.09 \times 10^{-19} \mathrm{~s}^{-1}\left[\frac{1.25 z}{\left(z^{2}+0.0324\right)^{1 / 2}}+0.58 z\right],
$$

where $z=(D / \mathrm{kpc}) \operatorname{sinb}$. The planar component is given by

$$
\frac{\dot{P}_{\mathrm{G}, \mathrm{p}}}{P}=-\cos b\left(\frac{\Theta_{0}^{2}}{c R_{0}}\right)\left(\cos \ell+\frac{\beta}{\sin ^{2} \ell+\beta^{2}}\right),
$$

where $\Theta_{0}=240 \mathrm{~km} \mathrm{~s}^{-1}$ is the Sun's galactocentric velocity, $R_{0}=8.34 \mathrm{kpc}$ is the Sun's galactocentric distance, $\beta=\left(D_{\mathrm{DM}} / R_{0}\right) \cos b-\cos \ell$, and $\ell$ is the Galactic longitude. 
Table 3

Derived Common Properties of GBNCC Pulsars

\begin{tabular}{|c|c|c|c|c|c|c|c|}
\hline PSR & $\begin{array}{l}P \\
\text { (s) }\end{array}$ & $\begin{array}{c}\dot{P} \\
\left(\mathrm{~s} \mathrm{~s}^{-1}\right)\end{array}$ & $\begin{array}{c}\tau_{\mathrm{c}} \\
(\mathrm{yr})\end{array}$ & $\begin{array}{l}B_{\text {surf }} \\
(\mathrm{G})\end{array}$ & $\begin{array}{c}\dot{E}_{\mathrm{rot}} \\
\left(\mathrm{erg} \mathrm{s}^{-1}\right)\end{array}$ & $\begin{array}{c}D_{\mathrm{DM}}^{\mathrm{NE} 2001} \\
(\mathrm{kpc})\end{array}$ & $\begin{array}{c}D_{\mathrm{DM}}^{\mathrm{YMW} 16} \\
(\mathrm{kpc})\end{array}$ \\
\hline J0054+6946 & $0.832911328744(4)$ & $7.194(8) \times 10^{-16}$ & $1.834(2) \times 10^{7}$ & $7.832(4) \times 10^{11}$ & $4.915(5) \times 10^{31}$ & 4.3 & 2.8 \\
\hline $\mathrm{J} 0058+4950$ & $0.99602345227(4)$ & $8.0(2) \times 10^{-16}$ & $1.97(5) \times 10^{7}$ & $9.0(1) \times 10^{11}$ & $3.20(7) \times 10^{31}$ & 2.8 & 2.6 \\
\hline $\mathrm{J} 0111+6624$ & $4.3018721007(3)$ & $8.4(2) \times 10^{-15}$ & $8.1(2) \times 10^{6}$ & $6.07(6) \times 10^{12}$ & $4.15(9) \times 10^{30}$ & 3.4 & 2.4 \\
\hline $\mathrm{J} 0137+6349$ & $0.717962726847(7)$ & $9.60(5) \times 10^{-16}$ & $1.185(6) \times 10^{7}$ & $8.40(2) \times 10^{11}$ & $1.024(5) \times 10^{32}$ & 44.3 & 9.1 \\
\hline $\mathrm{J} 0212+5222$ & $0.376386364060(2)$ & $6.599(1) \times 10^{-15}$ & $9.037(2) \times 10^{5}$ & $1.5946(1) \times 10^{12}$ & $4.8860(9) \times 10^{33}$ & 1.5 & 1.6 \\
\hline $\mathrm{J} 0325+6744$ & $1.36467876728(1)$ & $1.553(9) \times 10^{-15}$ & $1.393(8) \times 10^{7}$ & $1.473(4) \times 10^{12}$ & $2.41(1) \times 10^{31}$ & 2.3 & 1.9 \\
\hline $\mathrm{J} 0335+6623$ & $1.7619342474(2)$ & $4.81(9) \times 10^{-15}$ & $5.8(1) \times 10^{6}$ & $2.95(3) \times 10^{12}$ & $3.47(7) \times 10^{31}$ & 2.3 & 1.9 \\
\hline $\mathrm{J} 0358+4155$ & $0.2264843322519(2)$ & $1.458(1) \times 10^{-16}$ & $2.461(2) \times 10^{7}$ & $1.8388(9) \times 10^{11}$ & $4.956(5) \times 10^{32}$ & 1.6 & 1.5 \\
\hline $\mathrm{J} 0358+6627$ & $0.0915057807547(2)$ & $1.09(1) \times 10^{-17}$ & $1.33(1) \times 10^{8}$ & $3.20(2) \times 10^{10}$ & $5.62(5) \times 10^{32}$ & 2.2 & 1.9 \\
\hline $\mathrm{J} 0509+3801$ & $0.0765413487220(1)$ & $7.931(2) \times 10^{-18}$ & $1.5291(5) \times 10^{8}$ & $2.4929(4) \times 10^{10}$ & $6.982(2) \times 10^{32}$ & 1.9 & 1.6 \\
\hline $\mathrm{J} 0518+5416$ & $0.340202651599(6)$ & $1.676(3) \times 10^{-15}$ & $3.217(6) \times 10^{6}$ & $7.639(7) \times 10^{11}$ & $1.680(3) \times 10^{33}$ & 1.5 & 1.4 \\
\hline $\mathrm{J} 0612+3721$ & $0.44387136767(2)$ & $1.5(1) \times 10^{-16}$ & $4.7(4) \times 10^{7}$ & $2.6(1) \times 10^{11}$ & $6.8(6) \times 10^{31}$ & 1.2 & 1.1 \\
\hline J0738+6904 & $6.8276928023(5)$ & $2.697(4) \times 10^{-14}$ & $4.012(6) \times 10^{6}$ & $1.373(1) \times 10^{13}$ & $3.345(5) \times 10^{30}$ & 0.8 & 1.1 \\
\hline $\mathrm{J} 0740+6620$ & $0.0028857364104907(1)$ & $1.2206(7) \times 10^{-20}$ & $3.746(2) \times 10^{9}$ & $1.8990(5) \times 10^{8}$ & $2.005(1) \times 10^{34}$ & 0.7 & 0.9 \\
\hline $\mathrm{J} 0747+6646$ & $0.407700524409(4)$ & $6.78(3) \times 10^{-16}$ & $9.53(4) \times 10^{6}$ & $5.32(1) \times 10^{11}$ & $3.95(2) \times 10^{32}$ & 1.2 & 1.9 \\
\hline J0944+4106 & $2.22943126698(4)$ & $4.421(3) \times 10^{-15}$ & $7.990(6) \times 10^{6}$ & $3.176(1) \times 10^{12}$ & $1.575(1) \times 10^{31}$ & 0.8 & 2.7 \\
\hline $\mathrm{J} 1059+6459$ & $3.631169678(2)$ & $5.73(5) \times 10^{-15}$ & $1.003(9) \times 10^{7}$ & $4.62(2) \times 10^{12}$ & $4.73(4) \times 10^{30}$ & 0.8 & 1.9 \\
\hline $\mathrm{J} 1125+7819$ & $0.004201609117875(1)$ & $7.26(8) \times 10^{-21}$ & $9.2(1) \times 10^{9}$ & $1.77(1) \times 10^{8}$ & $3.87(4) \times 10^{33}$ & 0.6 & 0.8 \\
\hline $\mathrm{J} 1624+8643$ & $0.395763022038(2)$ & $2.55(1) \times 10^{-16}$ & $2.46(1) \times 10^{7}$ & $3.211(6) \times 10^{11}$ & $1.621(6) \times 10^{32}$ & 3.0 & 0.5 \\
\hline $\mathrm{J} 1628+4406$ & $0.18117848994714(5)$ & $1.9378(3) \times 10^{-17}$ & $1.4814(2) \times 10^{8}$ & $5.9952(4) \times 10^{10}$ & $1.2863(2) \times 10^{32}$ & 0.6 & 8.8 \\
\hline $\mathrm{J} 1641+8049$ & $0.0020211793846822(1)$ & $8.95(7) \times 10^{-21}$ & $3.58(3) \times 10^{9}$ & $1.361(5) \times 10^{8}$ & $4.28(3) \times 10^{34}$ & 1.7 & 2.1 \\
\hline $\mathrm{J} 1647+6608$ & $1.59979837535(3)$ & $7.84(2) \times 10^{-15}$ & $3.233(7) \times 10^{6}$ & $3.583(4) \times 10^{12}$ & $7.56(2) \times 10^{31}$ & 1.3 & 3.0 \\
\hline $\mathrm{J} 1710+4923$ & $0.00322022839856445(4)$ & $1.8211(2) \times 10^{-20}$ & $2.8017(3) \times 10^{9}$ & $2.4502(1) \times 10^{8}$ & $2.1530(2) \times 10^{34}$ & 0.7 & 0.5 \\
\hline $\mathrm{J} 1800+5034$ & $0.57837510115(1)$ & $1.19(5) \times 10^{-16}$ & $7.7(4) \times 10^{7}$ & $2.65(6) \times 10^{11}$ & $2.4(1) \times 10^{31}$ & 1.4 & 1.9 \\
\hline $\mathrm{J} 1815+5546$ & $0.42684382706(4)$ & $9(2) \times 10^{-17}$ & $7(1) \times 10^{7}$ & $2.0(2) \times 10^{11}$ & $4.8(8) \times 10^{31}$ & 50.0 & 25.0 \\
\hline $\mathrm{J} 1821+4147$ & $1.261857209133(9)$ & $1.7292(9) \times 10^{-15}$ & $1.1562(6) \times 10^{7}$ & $1.4946(4) \times 10^{12}$ & $3.398(2) \times 10^{31}$ & 2.5 & 4.4 \\
\hline $\mathrm{J} 1859+7654$ & $1.39372913521(2)$ & $5(2) \times 10^{-17}$ & $4(1) \times 10^{8}$ & $2.7(4) \times 10^{11}$ & $6(2) \times 10^{29}$ & 2.9 & 6.0 \\
\hline $\mathrm{J} 1923+4243$ & $0.59519285851(1)$ & $2.534(5) \times 10^{-15}$ & $3.721(8) \times 10^{6}$ & $1.243(1) \times 10^{12}$ & $4.75(1) \times 10^{32}$ & 3.2 & 4.7 \\
\hline $\mathrm{J} 1934+5219$ & $0.56844228297(3)$ & $8(1) \times 10^{-17}$ & $1.1(2) \times 10^{8}$ & $2.2(2) \times 10^{11}$ & $1.8(3) \times 10^{31}$ & 4.5 & 7.7 \\
\hline $\mathrm{J} 1938+6604$ & $0.022258801226038(2)$ & $1.95(3) \times 10^{-20}$ & $1.81(3) \times 10^{10}$ & $6.66(6) \times 10^{8}$ & $7.0(1) \times 10^{31}$ & 2.3 & 3.4 \\
\hline $\mathrm{J} 1941+4320$ & $0.840906468392(6)$ & $1.1472(5) \times 10^{-15}$ & $1.1614(5) \times 10^{7}$ & $9.938(2) \times 10^{11}$ & $7.617(3) \times 10^{31}$ & 4.4 & 6.5 \\
\hline $\mathrm{J} 1942+8106$ & $0.203558452868(1)$ & $3.68(6) \times 10^{-17}$ & $8.8(1) \times 10^{7}$ & $8.76(7) \times 10^{10}$ & $1.72(3) \times 10^{32}$ & 2.1 & 3.5 \\
\hline $\mathrm{J} 1954+4357$ & $1.38704116015(2)$ & $2.202(3) \times 10^{-15}$ & $9.98(1) \times 10^{6}$ & $1.768(1) \times 10^{12}$ & $3.258(4) \times 10^{31}$ & 7.1 & 5.3 \\
\hline $\mathrm{J} 1955+6708$ & $0.0085654062678141(8)$ & $1.259(4) \times 10^{-20}$ & $1.078(4) \times 10^{10}$ & $3.323(6) \times 10^{8}$ & $7.91(3) \times 10^{32}$ & 3.4 & 10.1 \\
\hline $\mathrm{J} 2001+4258$ & $0.719166137852(4)$ & $1.7327(2) \times 10^{-14}$ & $6.5762(8) \times 10^{5}$ & $3.5717(2) \times 10^{12}$ & $1.8390(2) \times 10^{33}$ & 3.3 & 3.8 \\
\hline $\mathrm{J} 2017+5906$ & $0.40347833490(2)$ & $2.22(1) \times 10^{-16}$ & $2.88(2) \times 10^{7}$ & $3.026(9) \times 10^{11}$ & $1.332(8) \times 10^{32}$ & 3.3 & 3.9 \\
\hline $\mathrm{J} 2027+7502$ & $0.515218649152(8)$ & $8.90(5) \times 10^{-16}$ & $9.17(5) \times 10^{6}$ & $6.85(2) \times 10^{11}$ & $2.57(1) \times 10^{32}$ & 1.0 & 0.8 \\
\hline $\mathrm{J} 2123+5434$ & $0.1388680725891(2)$ & $1.7(1) \times 10^{-19}$ & $1.27(7) \times 10^{10}$ & $5.0(1) \times 10^{9}$ & $2.6(1) \times 10^{30}$ & 2.1 & 1.8 \\
\hline $\mathrm{J} 2137+6428$ & $1.75098916326(7)$ & $3.43(4) \times 10^{-15}$ & $8.1(1) \times 10^{6}$ & $2.48(1) \times 10^{12}$ & $2.53(3) \times 10^{31}$ & 4.8 & 3.8 \\
\hline $\mathrm{J} 2208+4056$ & $0.63695739361(1)$ & $5.283(1) \times 10^{-15}$ & $1.9102(4) \times 10^{6}$ & $1.8561(2) \times 10^{12}$ & $8.071(2) \times 10^{32}$ & 1.0 & 0.8 \\
\hline $\mathrm{J} 2228+6447$ & $1.89298180355(5)$ & $6.7(3) \times 10^{-16}$ & $4.5(2) \times 10^{7}$ & $1.14(3) \times 10^{12}$ & $3.9(2) \times 10^{30}$ & 46.9 & 6.9 \\
\hline $\mathrm{J} 2241+6941$ & $0.85540126571(1)$ & $2.693(8) \times 10^{-15}$ & $5.03(1) \times 10^{6}$ & $1.536(2) \times 10^{12}$ & $1.699(5) \times 10^{32}$ & 2.9 & 2.5 \\
\hline $\mathrm{J} 2310+6706$ & $1.944788973(1)$ & $6(5) \times 10^{-17}$ & $5(4) \times 10^{8}$ & $3(1) \times 10^{11}$ & $2(3) \times 10^{29}$ & 3.5 & 2.7 \\
\hline $\mathrm{J} 2312+6931$ & $0.81337477832(2)$ & $6.3(1) \times 10^{-16}$ & $2.04(4) \times 10^{7}$ & $7.25(6) \times 10^{11}$ & $4.63(8) \times 10^{31}$ & 2.8 & 2.4 \\
\hline $\mathrm{J} 2351+8533$ & $1.01172719111(6)$ & $8.8(3) \times 10^{-16}$ & $1.83(7) \times 10^{7}$ & $9.5(2) \times 10^{11}$ & $3.3(1) \times 10^{31}$ & 1.9 & 2.6 \\
\hline
\end{tabular}

Note. Here $D_{\text {DM }}$ is calculated using the NE2001 Cordes \& Lazio (2002) or YMW16 (Yao et al. 2017) Galactic free electron density models, as indicated. A fractional uncertainty of $50 \%$ is not uncommon. Derived parameters have not been corrected for the Shklovskii effect. Here $\dot{E}$ is calculated assuming a moment of inertia $I=10^{45} \mathrm{gmcm}^{2}$. Values in parentheses are the $1 \sigma$ uncertainty in the last digit, calculated by propagating uncertainties in measured parameters reported by TEMPO.

The Shklovskii effect (Shklovskii 1970) is

$$
\frac{\dot{P}_{\mathrm{S}}}{P}=2.43 \times 10^{-21} \mathrm{~s}^{-1}\left(\frac{D_{\mathrm{DM}}}{\mathrm{kpc}}\right)\left(\frac{\mu}{10^{-3} \mathrm{yr}^{-1}}\right)^{2} .
$$

Note that in the case of PSRs J1125+7819, J1641+8049, and $\mathrm{J} 1710+4923$, the DM-inferred distance under one or both of the NE2001 and YMW16 models leads to values of $\dot{P}_{\mathrm{S}}$ larger than the observed $\dot{P}$. None of these pulsars show any evidence that they are being spun up via accretion, so in all three cases, $\dot{P}_{\text {int }}$ should be positive. Since $\left|\dot{P}_{\mathrm{G}}\right| \ll \dot{P}_{\mathrm{S}}$ in these cases, we can ignore the Galactic acceleration component and enforce $\dot{P}_{\mathrm{S}}<\dot{P}_{\text {obs }}$, thereby setting an upper limit on the distances (note that we do not use these distance limits to reestimate $\dot{P}_{\mathrm{G}}$ ). For PSR J1125+7819, $D<0.7 \mathrm{kpc}$ and $v_{\mathrm{t}}<104 \mathrm{~km} \mathrm{~s}^{-1}$; for PSR $\mathrm{J} 1641+8049, D<1.2 \mathrm{kpc}$ and $v_{\mathrm{t}}<230 \mathrm{~km} \mathrm{~s}^{-1}$; and for PSR $\mathrm{J} 1710+4923, D<0.5 \mathrm{kpc}$ and $v_{\mathrm{t}}<160 \mathrm{~km}, \mathrm{~s}^{-1}$.

The mean transverse velocity of our sample of MSPs is $\mu_{v}=152 \mathrm{~km} \mathrm{~s}^{-1}$ with a standard deviation of $\sigma_{v}=48 \mathrm{~km} \mathrm{~s}^{-1}$ using the NE2001 DM-inferred distances and $\mu_{v}=234 \mathrm{~km} \mathrm{~s}^{-1}$, $\sigma_{v}=143 \mathrm{~km} \mathrm{~s}^{-1}$ using the YMW16 DM-inferred distances 
Table 4

Coordinates and DMs of GBNCC Pulsars

\begin{tabular}{|c|c|c|c|c|c|c|c|}
\hline \multirow[t]{2}{*}{ PSR } & \multicolumn{3}{|c|}{ Measured } & \multicolumn{4}{|c|}{ Derived } \\
\hline & $\lambda(\operatorname{deg})$ & $\beta(\operatorname{deg})$ & $\mathrm{DM}\left(\mathrm{pc} \mathrm{cm}^{-3}\right)$ & $\alpha(\mathrm{J} 2000)$ & $\delta(\mathrm{J} 2000)$ & $\ell(\operatorname{deg})$ & $b(\mathrm{deg})$ \\
\hline J0054+6946 & $53.17957(2)$ & $55.915268(8)$ & $116.52(5)$ & $00^{\mathrm{h}} 54^{\mathrm{m}} 59^{\mathrm{s}} .109$ & $69^{\circ} 46^{\prime} 16^{\prime \prime} 9$ & 123.24077 & 6.90173 \\
\hline $\mathrm{J} 0058+4950$ & $35.9410(1)$ & $39.55191(6)$ & $66.953(7)$ & $00^{\mathrm{h}} 58^{\mathrm{m}} 09^{\mathrm{s}} .989$ & $49^{\circ} 50^{\prime} 26^{\prime \prime} 0$ & 124.04528 & -13.01663 \\
\hline $\mathrm{J} 0111+6624$ & $51.3828(4)$ & $52.3710(1)$ & $111.20(3)$ & $01^{\mathrm{h}} 11^{\mathrm{m}} 21^{\mathrm{s}} .862$ & $66^{\circ} 24^{\prime} 10^{\prime \prime} 9$ & 124.92791 & 3.60854 \\
\hline $\mathrm{J} 0137+6349$ & $52.48260(5)$ & 48.69309(3) & $285.50(6)$ & $01^{\mathrm{h}} 37^{\mathrm{m}} 13^{\mathrm{s}} .362$ & $63^{\circ} 49^{\prime} 34^{\prime \prime} 4$ & 127.95520 & 1.40191 \\
\hline $\mathrm{J} 0212+5222$ & $50.60763(2)$ & $36.42041(1)$ & $38.21(3)$ & $02^{\mathrm{h}} 12^{\mathrm{m}} 52^{\mathrm{s}} \cdot 136$ & $52^{\circ} 22^{\prime} 49^{\prime \prime} 5$ & 135.33111 & -8.51996 \\
\hline J0325+6744 & $69.66385(5)$ & $47.02620(3)$ & $65.28(5)$ & $03^{\mathrm{h}} 25^{\mathrm{m}} 05^{\mathrm{s}} .117$ & $67^{\circ} 44^{\prime} 59^{\prime \prime} 4$ & 136.71527 & 9.08929 \\
\hline $\mathrm{J} 0335+6623$ & $70.4110(3)$ & $45.3827(3)$ & $66.726(2)$ & $03^{\mathrm{h}} 35^{\mathrm{m}} 57^{\mathrm{s}} \cdot 077$ & $66^{\circ} 23^{\prime} 23^{\prime \prime} 6$ & 138.38339 & 8.58769 \\
\hline $\mathrm{J} 0358+4155$ & $66.154390(4)$ & $20.973526(6)$ & $46.325(1)$ & $03^{\mathrm{h}} 58^{\mathrm{m}} 03^{\mathrm{s}} \cdot 174$ & $41^{\circ} 55^{\prime} 19^{\prime \prime} 1$ & 156.11209 & -8.62040 \\
\hline $\mathrm{J} 0358+6627$ & $73.496599(8)$ & $44.753531(6)$ & $62.33(1)$ & $03^{\mathrm{h}} 58^{\mathrm{m}} 37^{\mathrm{s}} \cdot 926$ & $66^{\circ} 27^{\prime} 46^{\prime \prime} 6$ & 140.12843 & 10.06385 \\
\hline $\mathrm{J} 0509+3801$ & $79.7362277(8)$ & $15.030898(4)$ & 69.0794(9) & $05^{\mathrm{h}} 09^{\mathrm{m}} 31^{\mathrm{s}} .788$ & $38^{\circ} 01^{\prime} 18^{\prime \prime} 1$ & 168.27474 & -1.18699 \\
\hline $\mathrm{J} 0518+5416$ & $83.01300(5)$ & $31.09029(8)$ & $42.330(5)$ & $05^{\mathrm{h}} 18^{\mathrm{m}} 53^{\mathrm{s}} .198$ & $54^{\circ} 16^{\prime} 50^{\prime \prime} 0$ & 155.91644 & 9.55747 \\
\hline $\mathrm{J} 0612+3721$ & $92.6070(1)$ & $13.9507(6)$ & $39.270(6)$ & $06^{\mathrm{h}} 12^{\mathrm{m}} 44^{\mathrm{s}} .087$ & $37^{\circ} 21^{\prime} 40^{\prime \prime} 2$ & 175.44220 & 9.08013 \\
\hline $\mathrm{J} 0738+6904$ & $102.51871(7)$ & $46.69934(9)$ & $17.22(2)$ & $07^{\mathrm{h}} 38^{\mathrm{m}} 022^{\mathrm{s}} \cdot 61$ & $69^{\circ} 04^{\prime} 20^{\prime \prime} 1$ & 146.59345 & 29.37720 \\
\hline $\mathrm{J} 0740+6620$ & $103.7591384(1)$ & $44.1025059(1)$ & $14.92(2)$ & $07^{\mathrm{h}} 40^{\mathrm{m}} 45^{\mathrm{s}} .799$ & $66^{\circ} 20^{\prime} 33^{\prime \prime} 6$ & 149.72969 & 29.59937 \\
\hline $\mathrm{J} 0747+6646$ & $104.53950(2)$ & $44.69663(5)$ & $27.576(3)$ & $07^{\mathrm{h}} 47^{\mathrm{m}} 39^{\mathrm{s}} \cdot 689$ & $66^{\circ} 46^{\prime} 56^{\prime \prime} 8$ & 149.21764 & 30.28271 \\
\hline J0944+4106 & 134.006413(9) & $25.83992(3)$ & 21.41(3) & $09^{\mathrm{h}} 44^{\mathrm{m}} 18^{\mathrm{s}} .141$ & $41^{\circ} 06^{\prime} 04^{\prime \prime} 6$ & 180.43732 & 49.37512 \\
\hline $\mathrm{J} 1059+6459$ & $131.4687(7)$ & $51.9583(6)$ & $18.5(4)$ & $10^{\mathrm{h}} 59^{\mathrm{m}} 27^{\mathrm{s}} .511$ & $64^{\circ} 59^{\prime} 31^{\prime \prime} 8$ & 140.25104 & 48.19754 \\
\hline $\mathrm{J} 1125+7819$ & $115.6292886(9)$ & $62.4520225(4)$ & 11.219201 & $11^{\mathrm{h}} 25^{\mathrm{m}} 59^{\mathrm{s}} .851$ & $78^{\circ} 19^{\prime} 48^{\prime \prime} 7$ & 128.28875 & 37.89467 \\
\hline $\mathrm{J} 1624+8643$ & $93.79282(3)$ & $69.51940(1)$ & $46.43(2)$ & $16^{\mathrm{h}} 24^{\mathrm{m}} 032^{\mathrm{s}} .75$ & $86^{\circ} 43^{\prime} 13^{\prime \prime} 2$ & 119.92489 & 29.05717 \\
\hline $\mathrm{J} 1628+4406$ & $229.913199(1)$ & $64.4120718(2)$ & $7.32981(2)$ & $16^{\mathrm{h}} 28^{\mathrm{m}} 50^{\mathrm{s}} .313$ & $44^{\circ} 06^{\prime} 42^{\prime \prime} 6$ & 69.23851 & 43.61575 \\
\hline $\mathrm{J} 1641+8049$ & $101.8728674(9)$ & $74.8941763(2)$ & $31.08960(3)$ & $16^{\mathrm{h}} 41^{\mathrm{m}} 20^{\mathrm{s}} .843$ & $80^{\circ} 49^{\prime} 52^{\prime \prime} 9$ & 113.84003 & 31.76257 \\
\hline $\mathrm{J} 1647+6608$ & $174.9821(4)$ & $82.74687(3)$ & $22.55(7)$ & $16^{\mathrm{h}} 47^{\mathrm{m}} 32^{\mathrm{s}} \cdot 522$ & $66^{\circ} 08^{\prime} 22^{\prime \prime} 2$ & 97.18025 & 37.03415 \\
\hline $\mathrm{J} 1710+4923$ & $243.4177674(2)$ & $71.67561410(7)$ & $7.08493(2)$ & $17^{\mathrm{h}} 10^{\mathrm{m}} 04^{\mathrm{s}} .442$ & $49^{\circ} 23^{\prime} 11^{\prime \prime} 4$ & 75.92934 & 36.44891 \\
\hline $\mathrm{J} 1800+5034$ & $270.4263(1)$ & $74.01172(4)$ & $22.71(6)$ & $18^{\mathrm{h}} 00^{\mathrm{m}} 44^{\mathrm{s}} .372$ & $50^{\circ} 34^{\prime} 21^{\prime \prime} 7$ & 78.12962 & 28.43981 \\
\hline $\mathrm{J} 1815+5546$ & $281.2542(7)$ & $79.0649(2)$ & $58.999(7)$ & $18^{\mathrm{h}} 15^{\mathrm{m}} 05^{\mathrm{s}} .739$ & $55^{\circ} 46^{\prime} 23^{\prime \prime} 2$ & 84.31588 & 27.13337 \\
\hline $\mathrm{J} 1821+4147$ & $279.69363(3)$ & 65.03943(1) & $40.673(3)$ & $18^{\mathrm{h}} 21^{\mathrm{m}} 52^{\mathrm{s}} \cdot 346$ & $41^{\circ} 47^{\prime} 02^{\prime \prime} 5$ & 69.53713 & 22.90515 \\
\hline $\mathrm{J} 1859+7654$ & $72.6850(2)$ & $78.72083(4)$ & $47.25(7)$ & $18^{\mathrm{h}} 59^{\mathrm{m}} 36^{\mathrm{s}} .019$ & $76^{\circ} 54^{\prime} 55^{\prime \prime} 6$ & 108.36384 & 25.91076 \\
\hline $\mathrm{J} 1923+4243$ & $305.93303(4)$ & $63.58758(4)$ & $52.99(5)$ & $19^{\mathrm{h}} 23^{\mathrm{m}} 015^{\mathrm{s}} \cdot 24$ & $42^{\circ} 43^{\prime} 18^{\prime \prime} 6$ & 74.71528 & 12.64331 \\
\hline $\mathrm{J} 1934+5219$ & $320.8887(1)$ & $71.6218(1)$ & $71.9(1)$ & $19^{\mathrm{h}} 34^{\mathrm{m}} 23^{\mathrm{s}} .892$ & $52^{\circ} 19^{\prime} 57^{\prime \prime} 4$ & 84.49263 & 15.04604 \\
\hline $\mathrm{J} 1938+6604$ & $8.602915(1)$ & $80.1174609(2)$ & $41.2427(1)$ & $19^{\mathrm{h}} 38^{\mathrm{m}} 56^{\mathrm{s}} .919$ & $66^{\circ} 04^{\prime} 31^{\prime \prime} 7$ & 97.94564 & 20.03050 \\
\hline $\mathrm{J} 1941+4320$ & $313.54787(3)$ & $62.971984(8)$ & $79.361(8)$ & $19^{\mathrm{h}} 41^{\mathrm{m}} 58^{\mathrm{s}} .915$ & $43^{\circ} 20^{\prime} 06^{\prime \prime} 3$ & 76.84556 & 9.86437 \\
\hline $\mathrm{J} 1942+8106$ & $75.79591(5)$ & $74.12426(1)$ & $40.24(3)$ & $19^{\mathrm{h}} 42^{\mathrm{m}} 54^{\mathrm{s}} .669$ & $81^{\circ} 06^{\prime} 17^{\prime \prime} 3$ & 113.37123 & 24.82517 \\
\hline $\mathrm{J} 1954+4357$ & $318.69172(4)$ & $62.63881(2)$ & $130.30(5)$ & $19^{\mathrm{h}} 54^{\mathrm{m}} 038^{\mathrm{s}} .45$ & $43^{\circ} 57^{\prime} 37^{\prime \prime} 3$ & 78.52732 & 8.16863 \\
\hline $\mathrm{J} 1955+6708$ & $16.178385(1)$ & $78.7214729(2)$ & $57.1478(1)$ & $19^{\mathrm{h}} 55^{\mathrm{m}} 38^{\mathrm{s}} .764$ & $67^{\circ} 08^{\prime} 15^{\prime \prime} 1$ & 99.68213 & 18.93834 \\
\hline $\mathrm{J} 2001+4258$ & $320.10684(2)$ & $61.24332(2)$ & $54.93(3)$ & $20^{\mathrm{h}} 01^{\mathrm{m}} 010^{\mathrm{s}} .59$ & $42^{\circ} 58^{\prime} 06^{\prime \prime} 2$ & 78.27728 & 6.64261 \\
\hline $\mathrm{J} 2017+5906$ & $350.7866(2)$ & $72.89756(3)$ & $60.28(6)$ & $20^{\mathrm{h}} 17^{\mathrm{m}} 44^{\mathrm{s}} .555$ & $59^{\circ} 06^{\prime} 46^{\prime \prime} 9$ & 93.60825 & 12.90340 \\
\hline $\mathrm{J} 2027+7502$ & $51.5269(2)$ & $75.59357(2)$ & 11.71(1) & $20^{\mathrm{h}} 27^{\mathrm{m}} 23^{\mathrm{s}} \cdot 274$ & $75^{\circ} 02^{\prime} 29^{\prime \prime} 2$ & 108.36769 & 20.31252 \\
\hline $\mathrm{J} 2123+5434$ & $358.500835(3)$ & $63.277719(2)$ & $31.760(3)$ & $21^{\mathrm{h}} 23^{\mathrm{m}} 21^{\mathrm{s}} \cdot 681$ & $54^{\circ} 34^{\prime} 07^{\prime \prime} 7$ & 95.69847 & 3.09706 \\
\hline $\mathrm{J} 2137+6428$ & $20.1556(4)$ & $68.10698(6)$ & $106.0(3)$ & $21^{\mathrm{h}} 37^{\mathrm{m}} 20^{\mathrm{s}} .257$ & $64^{\circ} 28^{\prime} 41^{\prime \prime} 7$ & 103.85139 & 9.06360 \\
\hline $\mathrm{J} 2208+4056$ & $354.46060(4)$ & $47.91605(4)$ & 11.837(9) & $22^{\mathrm{h}} 08^{\mathrm{m}} 01^{\mathrm{s}} .991$ & $40^{\circ} 56^{\prime} 01^{\prime \prime} 8$ & 92.57410 & -12.11198 \\
\hline $\mathrm{J} 2228+6447$ & $27.9436(2)$ & $63.61524(4)$ & $193.6(2)$ & $22^{\mathrm{h}} 28^{\mathrm{m}} 40^{\mathrm{s}} .502$ & $64^{\circ} 47^{\prime} 19^{\prime \prime} 4$ & 108.46152 & 6.00724 \\
\hline $\mathrm{J} 2241+6941$ & $39.1473(1)$ & $65.08555(2)$ & $67.67(7)$ & $22^{\mathrm{h}} 41^{\mathrm{m}} 20^{\mathrm{s}} .295$ & $69^{\circ} 41^{\prime} 59^{\prime \prime} 7$ & 112.02560 & 9.63205 \\
\hline $\mathrm{J} 2310+6706$ & $37.3837(3)$ & $61.4356(4)$ & $97.7(2)$ & $23^{\mathrm{h}} 10^{\mathrm{m}} 42^{\mathrm{s}} .077$ & $67^{\circ} 06^{\prime} 52^{\prime \prime} 1$ & 113.35114 & 6.13557 \\
\hline $\mathrm{J} 2312+6931$ & $41.8523(2)$ & $62.62711(4)$ & $71.6(1)$ & $23^{\mathrm{h}} 12^{\mathrm{m}} 038^{\mathrm{s}} .93$ & $69^{\circ} 31^{\prime} 04^{\prime \prime} 0$ & 114.43546 & 8.29219 \\
\hline $\mathrm{J} 2351+8533$ & $78.8764(3)$ & $66.3376(2)$ & $38.5(4)$ & $23^{\mathrm{h}} 51^{\mathrm{m}} 03^{\mathrm{s}} .261$ & $85^{\circ} 33^{\prime} 20^{\prime \prime} 7$ & 121.67724 & 22.83186 \\
\hline
\end{tabular}

Note. Ecliptic coordinates use the IERS2010 value of the obliquity of the ecliptic referenced to J2000 (Capitaine et al. 2003). Values in parentheses are the $1 \sigma$ uncertainty in the last digit as reported by TEMPO.

(here we use the upper limits on $v_{\mathrm{t}}$ as appropriate). Uncertainty in the DM-inferred distances makes a precise measurement of transverse velocity difficult. With this caveat in mind, the velocities that we estimate are somewhat higher than those found by other authors, though still within one to two standard deviations: Toscano et al. (1999) found $\mu_{v}=85 \mathrm{~km} \mathrm{~s}^{-1}, \sigma_{v}=13 \mathrm{~km} \mathrm{~s}^{-1}$ for a sample of 13 MSPs; Hobbs et al. (2005) found $\mu_{v}=87 \mathrm{~km} \mathrm{~s}^{-1}, \sigma_{v}=$ $13 \mathrm{~km} \mathrm{~s}^{-1}$ for a sample of 35 MSPs; and Gonzalez et al. (2011) found $\mu_{v}=88 \mathrm{~km} \mathrm{~s}^{-1}, \sigma_{v}=12 \mathrm{~km} \mathrm{~s}^{-1}$ for a sample of five MSPs.

\subsection{Discussions of Individual Systems}

\subsubsection{PSR J0509+3801: A New DNS System}

PSR J0509+3801 is part of a highly eccentric binary system with $P_{\mathrm{b}}=9.11 \mathrm{hr}$ and $e=0.586$. Early in our timing campaign, we measured a significant change in the longitude of periastron, $\dot{\omega}=3.031(2)^{\circ} \mathrm{yr}^{-1}$. In GR, $\dot{\omega}$ is related to the total system mass, and our measured value implies $M_{\text {tot }}=2.81 M_{\odot}$. Additional timing observations resulted in the measurement of the amplitude of the Einstein delay due to gravitational redshift and time dilation, $\gamma=0.0046(3) \mathrm{s}$. With 
Table 5

ELL1 Binary Parameters of GBNCC Pulsars

\begin{tabular}{|c|c|c|c|c|}
\hline Parameter & $\mathrm{J} 0740+6620$ & $\mathrm{~J} 1125+7819$ & $\mathrm{~J} 1641+8049$ & $\mathrm{~J} 1938+6604$ \\
\hline \multicolumn{5}{|c|}{ Measured Parameters } \\
\hline$P_{\mathrm{B}}$ (days) & $4.766944616(3)$ & $15.35544590(2)$ & $0.0908739634(1)$ & $2.467162727(1)$ \\
\hline$a \sin i / c(\mathrm{~s})$ & $3.977556(1)$ & $12.1924288(7)$ & $0.0640793(3)$ & $8.950738(1)$ \\
\hline$\epsilon_{1}$ & $-5.6(4) \times 10^{-6}$ & $-1.28(1) \times 10^{-5}$ & $0.000111(8)$ & $4.8(3) \times 10^{-6}$ \\
\hline$\epsilon_{2}$ & $-2.0(2) \times 10^{-6}$ & $1.0(1) \times 10^{-6}$ & $-5.4(6) \times 10^{-5}$ & $-2.81(3) \times 10^{-5}$ \\
\hline$e$ & $5.9(4) \times 10^{-6}$ & $1.29(1) \times 10^{-5}$ & $0.000123(8)$ & $2.85(3) \times 10^{-5}$ \\
\hline$\omega(\operatorname{deg})$ & $1.23(4)$ & $-1.495(8)$ & $-1.12(5)$ & $-0.17(1)$ \\
\hline$f_{\mathrm{M}}\left(M_{\odot}\right)$ & $0.002973387(3)$ & $0.008253317(1)$ & $3.42102(5) \times 10^{-5}$ & $0.12649217(6)$ \\
\hline$M_{\mathrm{c}, \min }\left(M_{\odot}\right)$ & 0.2 & 0.29 & 0.04 & 0.87 \\
\hline
\end{tabular}

Note. All timing models presented here use the ELL1 binary model, which is appropriate for low-eccentricity orbits. Binary parameters for the relativistic binary PSR J0509+3801 are shown in Table 7. Values in parentheses are the $1 \sigma$ uncertainty in the last digit as reported by TEMPO.

Table 6

Proper Motions and Kinematic Corrections for Five GBNCC Pulsars

\begin{tabular}{|c|c|c|c|c|c|c|c|c|c|}
\hline PSR & $\begin{array}{c}\mu_{\lambda} \\
\left(\operatorname{mas~yr}^{-1}\right)\end{array}$ & $\begin{array}{c}\mu_{\beta} \\
\left(\operatorname{mas~yr}^{-1}\right)\end{array}$ & $\begin{array}{l}D_{\mathrm{DM}} \\
(\mathrm{kpc})\end{array}$ & $\begin{array}{c}v_{\mathrm{t}} \\
\left(\mathrm{km} \mathrm{s}^{-1}\right)\end{array}$ & $\left(10^{-21}\right)$ & $\left.\dot{P}_{\mathrm{S}}{ }_{\left(10^{-21}\right.}\right)$ & $\begin{array}{c}B_{\text {surf }} \\
\left(10^{8} \mathrm{G}\right)\end{array}$ & $\begin{array}{c}\tau_{\mathrm{c}} \\
(\mathrm{Gyr})\end{array}$ & $\begin{array}{c}\dot{E} \\
\left(10^{33} \mathrm{erg} \mathrm{s}^{-1}\right)\end{array}$ \\
\hline \multirow[t]{2}{*}{$\mathrm{J} 0740+6620$} & $3.2(4)$ & $-33.7(6)$ & $0.7^{\mathrm{a}}$ & $112^{\mathrm{a}}$ & $-0.14^{\mathrm{a}}$ & $5.6^{\mathrm{a}}$ & $1.4^{\mathrm{a}}$ & $6.8^{\mathrm{a}}$ & $11^{\mathrm{a}}$ \\
\hline & & & $0.9^{\mathrm{b}}$ & $144^{\mathrm{b}}$ & $-0.13^{\mathrm{b}}$ & $7.2^{\mathrm{b}}$ & $1.2^{\mathrm{b}}$ & $8.9^{\mathrm{b}}$ & $8.4^{\mathrm{b}}$ \\
\hline $\mathrm{J} 1125+7819$ & & & $0.8^{\mathrm{b}}$ & $121^{\mathrm{b}}$ & $-0.44^{\mathrm{b}}$ & $8.4^{\mathrm{b}}$ & $\ldots$ & $\ldots$ & $\cdots$ \\
\hline \multirow{2}{*}{$\mathrm{J} 1641+8049$} & $-11(1)$ & $37(3)$ & $1.7^{\mathrm{a}}$ & $311^{\mathrm{a}}$ & $-0.32^{\mathrm{a}}$ & $12^{\mathrm{a}}$ & $\cdots$ & $\cdots$ & $\cdots$ \\
\hline & & & $2.1^{\mathrm{b}}$ & $384^{\mathrm{b}}$ & $-0.35^{\mathrm{b}}$ & $15^{\mathrm{b}}$ & $\ldots$ & $\ldots$ & $\ldots$ \\
\hline \multirow[t]{2}{*}{$\mathrm{J} 1955+6708$} & $-3(1)$ & $10(2)$ & $3.4^{\mathrm{a}}$ & $168^{\mathrm{a}}$ & $-2.2^{\mathrm{a}}$ & $2.9^{\mathrm{a}}$ & $3.2^{\mathrm{a}}$ & $11^{\mathrm{a}}$ & $0.75^{\mathrm{a}}$ \\
\hline & & & $10.1^{\mathrm{b}}$ & $500^{\mathrm{b}}$ & $-2.9^{\mathrm{b}}$ & $8.6^{\mathrm{b}}$ & $2.5^{\mathrm{b}}$ & $20^{\mathrm{b}}$ & $0.43^{\mathrm{b}}$ \\
\hline
\end{tabular}

Notes. Values of $B_{\text {surf }}, \tau_{\mathrm{c}}$, and $\dot{E}$ that have been corrected for the Shklovskii effect are shown for pulsars where $\dot{P}_{\mathrm{S}}<\dot{P}_{\text {obs }}$.

a These values use the NE2001 (Cordes \& Lazio 2002) DM-inferred distance.

b These values use the YMW16 (Yao et al. 2017) DM-inferred distance.

the measurement of two post-Keplerian parameters, we were able to measure the masses of both PSR J0509+3801 and its companion within the framework of GR. Using the DDGR timing model, which uses the masses as free parameters, we find $M_{\mathrm{p}}=1.34(8) M_{\odot}$ and $M_{\mathrm{c}}=1.46(8) M_{\odot}$. Table 7 gives our complete timing solution. The high companion mass and eccentricity lead us to classify this as a new DNS system.

We also performed a separate Bayesian analysis of the masses. The two-dimensional probability map was computed using a $\chi^{2}$ grid method and the best-fit timing solution, where the Shapiro delay parameters are held fixed at each mass-mass coordinate while all other parameters are allowed to float freely when using TEMPO and the current TOA data set. We then used the procedure outlined by Splaver et al. (2002) to compute probability densities from the $\chi^{2}$ grid and then marginalized over each mass coordinate to obtain one-dimensional probability distribution functions (PDFs) for the pulsar and companion masses. We finally computed the equal-tailed, $68.3 \%$ credible intervals and median values of the neutron star masses from these PDFs (see Figure 2). From this analysis, we obtain estimates of $M_{\mathrm{p}}=1.36(8) M_{\odot}$ and $M_{\mathrm{c}}=1.45(8) M_{\odot}$. These estimates and the credible intervals are consistent with the uncertainties determined by the least-squares fit obtained from TEMPO.

The Shapiro delay cannot be measured in this system with the current data presented in this work, since the rms timing residual for J0509+3801 exceeds the typical amplitudes of the relativistic signal. However, the neutron star masses are estimated using two post-Keplerian (PK) measurements under the assumption that GR is correct and without any consideration of the binary mass function. We therefore use the mass function and the two mass constraints to estimate the inclination angle and find that $i=33_{-2}^{+2 \circ}$ or $i=147_{-2}^{+2 \circ}$. The two inclination estimates are allowed, since the mass function depends on $\sin i$ only and therefore yields no constraint on the sign of $\cos i$.

The masses of both stars and their mass ratio are similar to those in most other DNS systems (Kiziltan et al. 2013).

\subsubsection{PSR J0740+6620: Constraints on Pulsar and Companion Mass}

We report here a weak, $2 \sigma$ measurement of the Shapiro delay in PSR J0740+6620. We find best-fit values of the Shapiro range and shape parameters of $r=1.0(3) \mu \mathrm{s}$ and $s=1.00017(99)$ when using the ELL1 model, which is a 


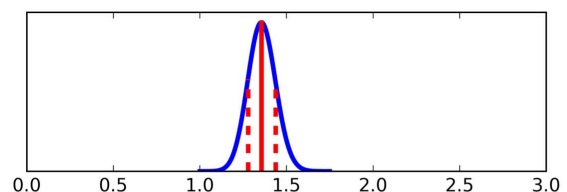

$\mathrm{J} 0509+3801$
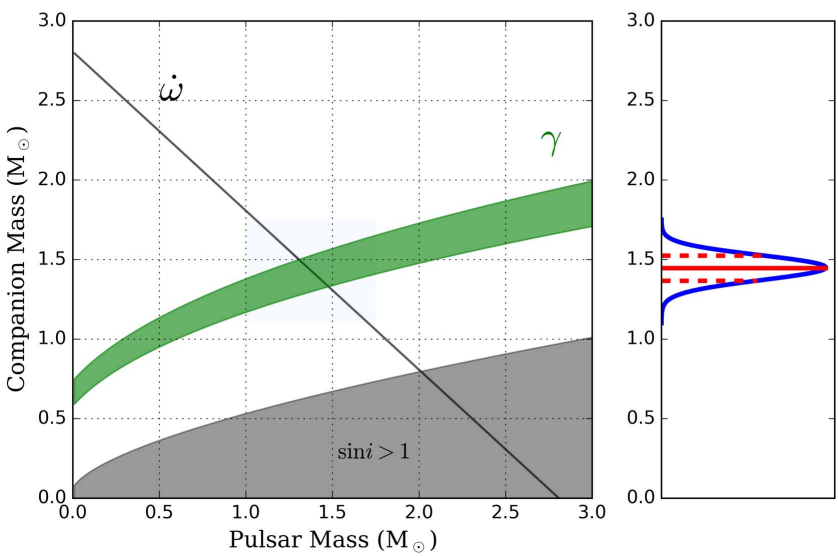

Figure 2. Estimates of the posterior probability density and marginalized distribution functions (blue curves) for mass estimates of the PSR J0509+3801 system. The regions of allowed masses for individual PK parameters are shown as color-shaded curves in the density map; the thickness of each color-shaded curve represents the $1 \sigma$ uncertainty determined by TEMPO (note that the uncertainties on $\dot{\omega}$ are too small to be visible on this scale). The red solid lines are the median values for each component mass, and the red dashed lines represent the edges of the $68.3 \%$ credible intervals.

Table 7

Binary Parameters of PSR J0509+3801

\begin{tabular}{ll}
\hline \hline Parameter & Value \\
\hline & \multicolumn{1}{c}{ Measured Parameters } \\
\hline$P_{\mathrm{b}}($ days $)$ & \\
$a \sin i / c(\mathrm{~s})$ & $0.379583785(3)$ \\
$T_{0}(\mathrm{MJD})$ & $2.0506(3)$ \\
$e$ & $56075.412714(3)$ \\
$\omega(\operatorname{deg})$ & $0.586400(6)$ \\
$M_{\mathrm{tot}}\left(M_{\odot}\right)$ & $127.77(1)$ \\
$M_{\mathrm{c}}\left(M_{\odot}\right)$ & $2.805(3)$ \\
\hline
\end{tabular}

Derived Parameters

\begin{tabular}{lc}
\hline$M_{\mathrm{p}}\left(M_{\odot}\right)$ & $1.34(8)$ \\
$f_{\mathrm{M}}\left(M_{\odot}\right)$ & $0.06425(3)$ \\
$\dot{\omega}\left(\operatorname{deg} \mathrm{yr}^{-1}\right)$ & 3.031 \\
$\gamma(\mathrm{s})$ & 0.0046 \\
$\dot{P}_{\mathrm{b}}\left(10^{-12}\right)$ & -1.39 \\
$\sin i$ & 0.55 \\
\hline
\end{tabular}

Note. Values in parentheses are the $1 \sigma$ uncertainty in the last digit as reported by TEMPO.

theory-independent model. Within the framework of GR, the Shapiro parameters become $r=T_{\odot} M_{\mathrm{c}}$, implying $M_{\mathrm{c}}=0.21(6)$, and $s=\sin i$. The best-fit value of $s$ is $1 \sigma$, consistent with $i<90^{\circ}$, but includes a nonphysical range. A dedicated campaign to observe PSR J0740+6620 near conjunction, when the Shapiro delay is maximum, will be the subject of a future study, but our current results are consistent with a nearly edge-on system and an $\sim 0.2 M_{\odot}$ companion.

We can constrain the companion mass along independent lines of reasoning as well. The low eccentricity and few-day orbital period of PSR J0740+6620 are consistent with expectations for an $\mathrm{He}$ WD companion. A well-defined relationship is observed between $P_{\mathrm{b}}$ and $M_{\mathrm{c}}$ in such systems (Tauris \& Savonije 1999; Istrate et al. 2016) and, in the case of PSR J0740+6620, predicts a companion mass $\sim 0.2 M_{\odot}$. We can also calculate the minimum companion mass by assuming $i=90^{\circ}$ and $M_{\mathrm{p}}=1.4 M_{\odot}$ and find $M_{\mathrm{c}, \min }=0.2 M_{\odot}$. Both of these values are consistent with the tentative measurement of $r$ and an inclination angle close to $90^{\circ}$ for a pulsar mass of 1.4 $M_{\odot}$. We therefore conclude that the pulsar's companion is an He WD and that the pulsar mass is close to the canonical value.

\subsubsection{PSR J1938+6604: An IMBP}

PSR J1938+6604 is a partially recycled binary pulsar with $P=22 \mathrm{~ms}$. The minimum companion mass assuming $M_{\mathrm{p}}=1.4 M_{\odot}$ and $i=90^{\circ}$ is $0.87 M_{\odot}$. Motivated by the potentially high companion mass, we conducted a campaign to measure Shapiro delay in PSR J1938+6604 using the GBT. We observed the pulsar for 6 hours around conjunction and for 2-4 hours at other select orbital phases where the measurable Shapiro delay signature is predicted to be at a local maximum. Our campaign totaled $21 \mathrm{hr}$ and was conducted at a center frequency of $1.4 \mathrm{GHz}$ using coherent dedispersion (see Table 1 for details). However, we were unable to detect Shapiro delay.

Our nondetection implies that the system is not highly inclined $\left(i \lesssim 80^{\circ}\right)$, and if we assume $M_{\mathrm{p}}=1.4 M_{\odot}$, the companion mass limit is $M_{\mathrm{c}}>0.88 M_{\odot}$. We see no evidence for eclipses, variations in DM, or changes in orbital period, and we find no optical companion in the Digital Sky Survey or Panoramic Survey Telescope and Rapid Response System (Pan-STARRS) archives, so the companion is unlikely to be a main-sequence or giant star. The eccentricity of the system is measurable and very low $\left(e=2.8 \times 10^{-5}\right)$, unlike DNS systems. PSR J1938+6620 is therefore most likely an IMBP (Camilo et al. 2001) with a CO/ONeMg WD companion.

\subsubsection{PSR J1641+8049: A Pulsar in a Black Widow Binary System}

PSR J1641+8049 is a fast MSP that exhibits eclipses and has a very low-mass companion $\left(M_{\mathrm{c}, \min }=0.04 M_{\odot}\right)$, making it a member of the black widow class of binary pulsars. In black widow systems, an energetic MSP ablates its companion, forming a low-mass remnant and perhaps eventually an isolated MSP (Fruchter et al. 1988; Phinney et al. 1988). Although we never observed ingress and egress for the same eclipse, based on pulsar timing, the eclipses lasted from orbital phases $\approx 0.205$ to 0.355 at $350 \mathrm{MHz}$, or about 20 minutes. The TOAs affected by excess DM near ingress and egress were not included in our timing analysis.

Since black widows have high spin-down luminosities, they are commonly detected in gamma rays (e.g., Ransom et al. 2011; Wu et al. 2012; Espinoza et al. 2013; Camilo et al. 2015). The upper limit on $\dot{E}$ for PSR J1641+8049 is in the middle of the sample of 51 publicly listed Fermi-detected MSPs $^{29}$ with measured $\dot{E}$ and distances listed in the ATNF pulsar catalog. We searched for pulsations in Fermi Large Area Telescope (LAT) events by downloading all events around a $3^{\circ}$ region of interest centered on the timing position reported in Table 4, recorded between MJDs 54802.65 and $57857.5,{ }^{30}$ and in an

\footnotetext{
${ }^{29}$ https://confluence.slac.stanford.edu/display/GLAMCOG/Public+List + of +LAT-Detected+Gamma-Ray+Pulsars

${ }^{30}$ Mission elapsed times 249925417-513863184.
} 


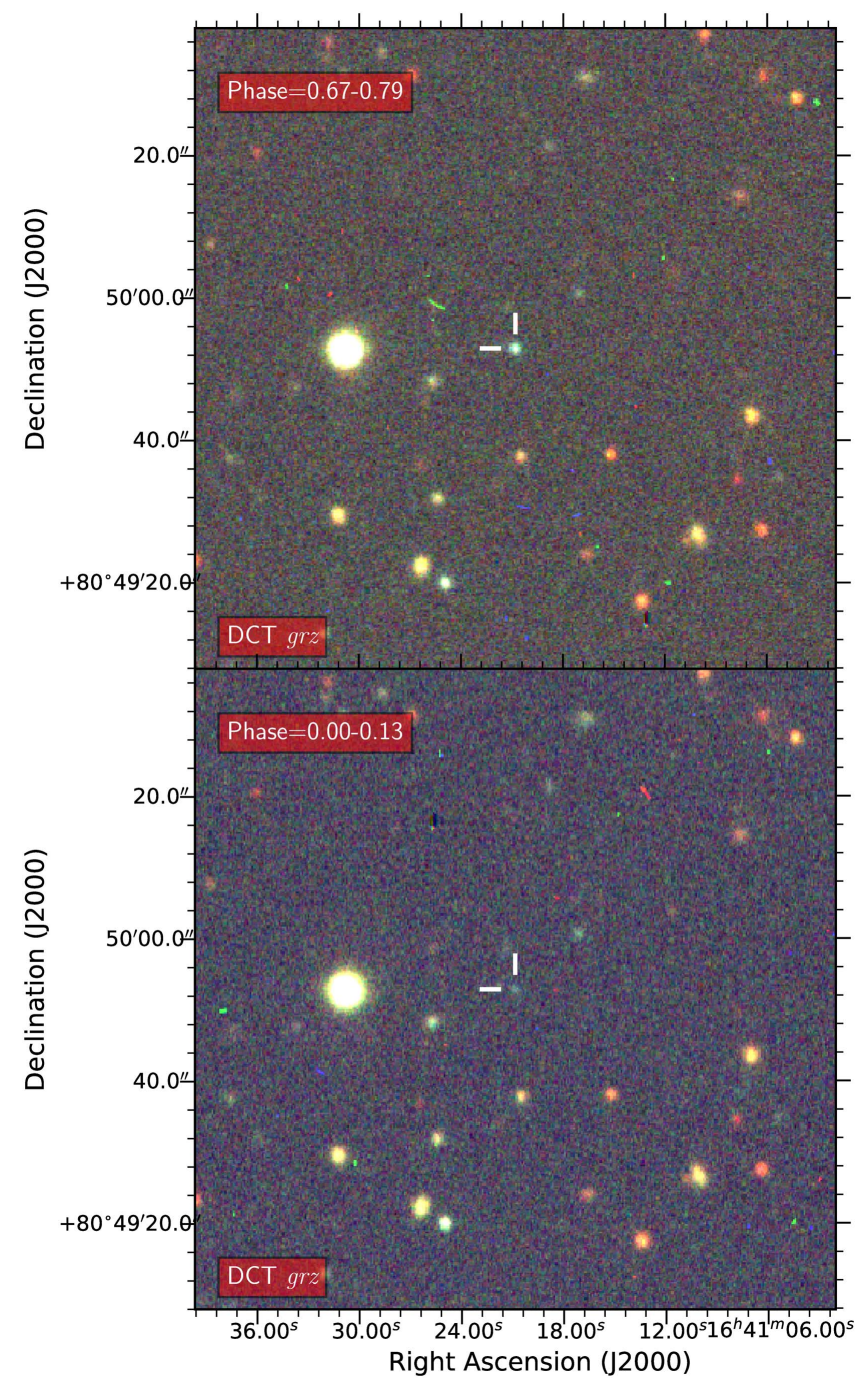

Figure 3. Composite rgb images of PSR J1641+8049, taken with the DCT's LMI at two different orbital phases: $0.67-0.79$ (close to photometric maximum) at the top and $0.00-0.13$ (close to photometric minimum) at the bottom. The composites are made from the $g, r$, and $z$ filters. The position of PSR J1641 +8049 is indicated with the ticks toward the center; the astrometric uncertainties are dominated by the astrometric calibration of the optical images, which has uncertainties of roughly $0 .{ }^{\prime \prime}$. The images are 1.5 on a side, with north up and east to the left.

energy range of 200--30, $000 \mathrm{keV}$. We used the fermiphase routine of the $\mathrm{PINT}^{31}$ pulsar timing and data analysis package to read the Fermi events, compute a pulse phase for each event, and calculate the $H$ statistic (de Jager et al. 1989) of the resulting light curve. This results in $H=3.26$, corresponding to an equivalent Gaussian $\sigma=1.10$; i.e., no significant pulsations are detected. It is plausible that the Shklovskii correction for PSR $\mathrm{J} 1641+8049$ is large and that the true $\dot{E}$ is much lower than the nominal value, explaining our nondetection.

\subsubsection{Optical Observations of the PSR J1641+8049 System}

We detected a faint optical counterpart to PSR J1641+8049 by examining the stacked images of the Pan-STARRS $3 \pi$ survey data release 1 (PS1; Chambers et al. 2016). It was only

\footnotetext{
31 http://nanograv-pint.readthedocs.io/en/latest/
}

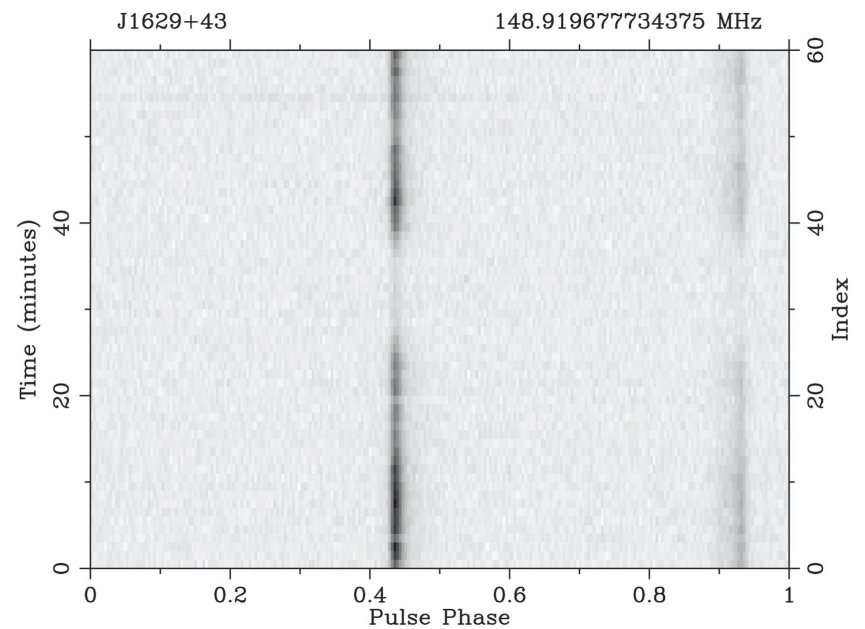

Figure 4. Phase-time plot of J1628+4406 from MJD 56639 as observed by LOFAR. The pulsar experiences a significant drop in flux for 15 minutes but does not appear to change modes. No other similar events were observed.

visible in the $r$ and $i$ bands and was not listed in the photometric catalogs. Therefore, we determined the rough photometry ourselves using the images and SExtractor (Bertin \& Arnouts 1996), finding $r=24.0 \pm 0.3$ and $i=$ $23.2 \pm 0.2$

To improve the photometry, we observed the field with the $4.3 \mathrm{~m}$ Discovery Channel Telescope (DCT) in Happy Jack, Arizona, using the Large Monolith Imager (LMI). Five $300 \mathrm{~s}$ observations in each of the $g, r, i$, and $z$ filters $(487,622,755$, and $868 \mathrm{~nm}$, respectively) were taken on 2017 March 16 from 08:58 to 10:42 UTC, spanning almost $2 \mathrm{hr}$, or slightly less than one orbit. Standard CCD reduction techniques (e.g., bias subtraction, flat fielding) were applied using a custom IRAF pipeline. Individual exposures were astrometrically aligned with respect to reference stars from the Sloan Digital Sky Survey (Ahn et al. 2014) using SCAMP (Bertin 2006). Composite rgb images near photometric maximum and minimum are shown in Figure 3. We calibrated the photometry using SExtractor and the PS1 catalog as a reference, with roughly 40 unsaturated stars per image per filter. The observation times were corrected to the solar system barycenter using PINT. We find a significant amount of photometric variation over the course of one orbit, with $r$ ranging from 21.7 to $>24.7$, and similar variability in the other filters. We also observed this system between 2017 March and April with the Sinistro camera on the $1 \mathrm{~m}$ telescope at the McDonald Observatory. There were 18 observations using the $r^{\prime}$ filter and 16 using $i^{\prime}$, all using $500 \mathrm{~s}$ exposures. We detected the system on 2017 March 19 at an orbital phase of $\phi_{\mathrm{B}} \approx 0.62$ (radio convention, where the ascending node marks $\phi_{\mathrm{B}}=0$ ) with $r^{\prime}=21.73 \pm 0.31$ and again on 2017 March 21 at $\phi_{\mathrm{B}} \approx 0.65$ with $r^{\prime}=21.38 \pm 0.23$ and $i^{\prime}=21.57 \pm 0.46$. All other observations resulted in nondetections.

Based on positional agreement and strong photometric variability tied to the orbital period, we can be certain that we have identified the optical counterpart of PSR J1641+8049. Future observations and analysis will enable us to determine the range of radii and effective temperatures for the companion and use them to constrain the mass of the pulsar and inclination of the binary system (e.g., Antoniadis et al. 2013). 


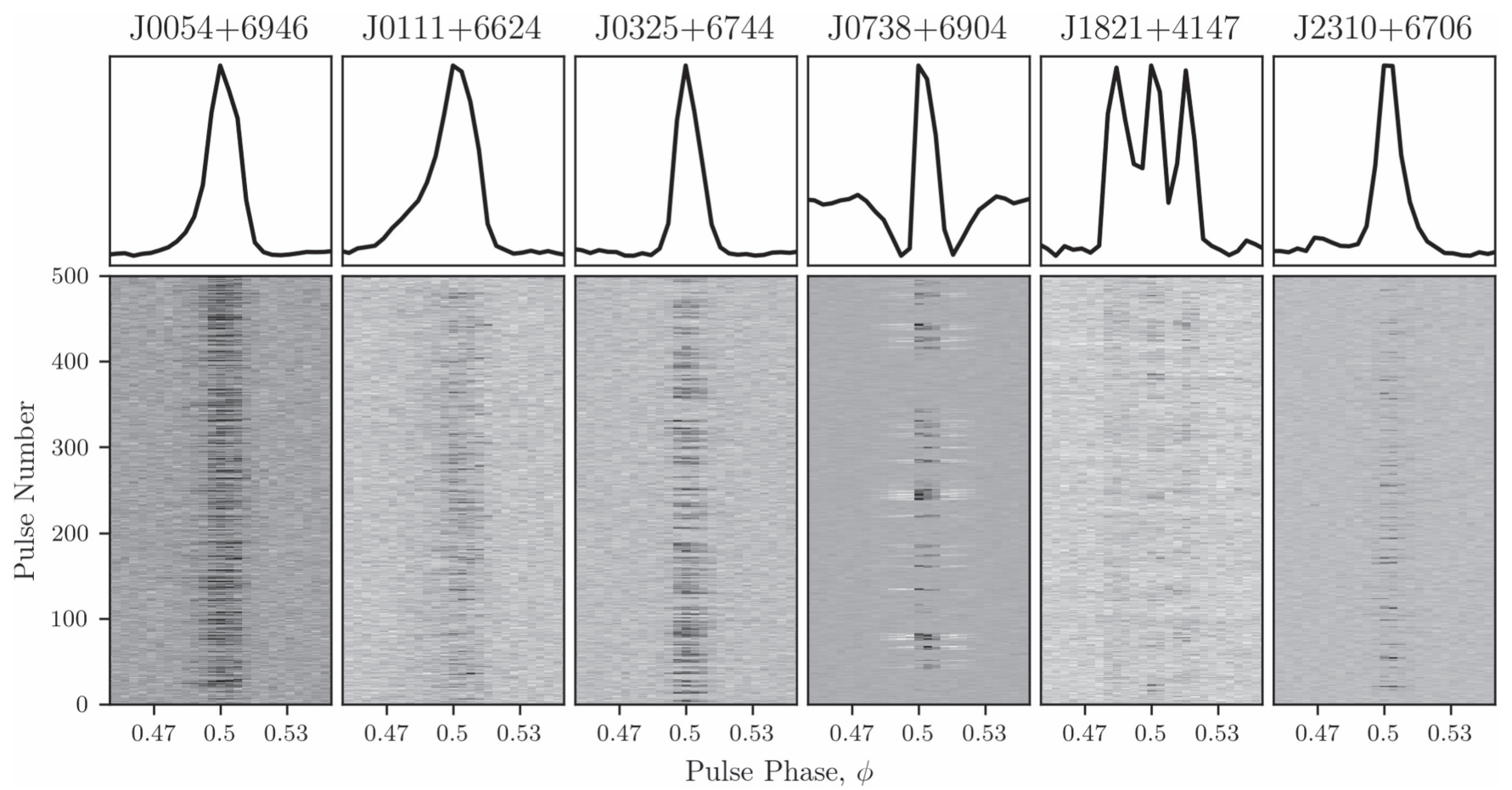

Figure 5. Preliminary single-pulse analysis for six GBNCC nulling pulsars with existing timing solutions. All data were taken at $820 \mathrm{MHz}$. For each source, 500 pulses are plotted in the bottom panels to show nulling behavior; folded profiles are shown in the top panels, corresponding to the same duration of pulse phase $(0.45<\phi<0.55)$. The apparent drop in flux on either side of the profile in PSR J0738+6904 is an artifact affecting the subbanded data of bright pulsars. Since it is clear when the pulsar is in an $\mathrm{ON}$ or OFF state, we do not expect it to bias the results of our nulling analysis.

\subsubsection{PSR J1628+4406: A New Mode-changing Pulsar}

PSR J1628+4406 has two emission modes, distinguished by differences in the amplitude of its pulse profile components (see Figure 9). In the first mode, the main pulse has two components with similar amplitudes (the leading component, i.e., leftward in Figure 9, is somewhat weaker than the trailing component), and there is a strong interpulse separated by $180^{\circ}$ in pulse phase from the trailer component of the main pulse. This general description of Mode 1 holds in both the 350 and $820 \mathrm{MHz}$ bands. In the $148 \mathrm{MHz}$ band observed with LOFAR, the leading component of the main pulse is blended with the trailing component, but, given the asymmetry in the profile, the leading component seems to be much weaker. In the second mode, the amplitude of the leading component of the main pulse is greatly reduced and the interpulse nearly vanishes, though it is still visible when several observations in this mode are summed together. Each component remains at the same pulse phase in both modes.

We observed PSR J1628+4406 for a total of 48,091 s during our timing campaign, split between the $148 \mathrm{MHz}$ LOFAR band (where the vast majority of the observations were made) and the 350 and $820 \mathrm{MHz}$ bands of the GBT. Of this, the pulsar spent 33,516 s (70\%) in Mode 1, while the remaining time $(14,574 \mathrm{~s} ; 30 \%)$ was spent in Mode 2 . We never witnessed a transition between modes during an observation, but we did observe a single event in which the pulsar significantly dropped in flux across both profile components for approximately 15 minutes before recovering to its prior state (see Figure 4). There is no record of an instrumental failure that would account for this drop in flux, nor was there an indication of solar activity that might cause ionospheric changes that would impact the observation at this level, so the flux change would appear to be a genuine phenomenon in this pulsar.

\subsubsection{PSR J2123+5434: A 138 ms Pulsar with a Low Magnetic Field}

At the completion of our timing program, our timing solution for PSR J2123+5434 did not constrain $\dot{P}$. Therefore, we obtained additional observations for this pulsar through our survey program about $3 \mathrm{yr}$ after the initial program, giving us a total time span of $4 \mathrm{yr}$. These additional observations enabled a $\dot{P}$ measurement of $1.7(1) \times 10^{-19}$, implying a surface magnetic field of $B_{\text {surf }}=5.0(1) \times 10^{9} \mathrm{G}$. These values are smaller than any known pulsar with a period $>100 \mathrm{~ms}$ that has a constrained $\dot{P}$ measurement. Figure 1 shows the location of PSR J2123 +5434 in the $P-\dot{P}$ plane in relation to the pulsars in the ATNF pulsar catalog. The expected change in $\dot{P}$ due to Galactic motion for PSR J2123+5434 (using the DM-derived distance of $2.1 \mathrm{kpc}$; Cordes \& Lazio 2002) is about $-2.3 \times$ $10^{-20}$. After applying this correction, PSR J2123+5434 remains an outlier in comparison to the typical pulsar. A potential explanation for PSR J2123+5434's anomalously low $\dot{P}$ is that it was partially recycled by a high-mass companion star that later underwent a supernova, disrupting the system. Alternatively, the pulsar may be in a very wide binary, in which case we could be observing orbital phases in which the pulsar is accelerating toward the Earth, inducing a Doppler $\dot{P}<0$ (e.g., PSR J1024-0719; Bassa et al. 2016; Kaplan et al. 2016). We examined archival infrared and optical data at the timing position of $\mathrm{J} 2123+5434$ but did not identify a counterpart at any wavelength.

\section{Nulling and Intermittent Pulsars}

Given the rarity of the phenomenon, nulling studies typically characterize pulsars by their "nulling fractions" (NFs; the fraction of time spent in a null state) and look for correlations between NF and other measured/derived parameters like 

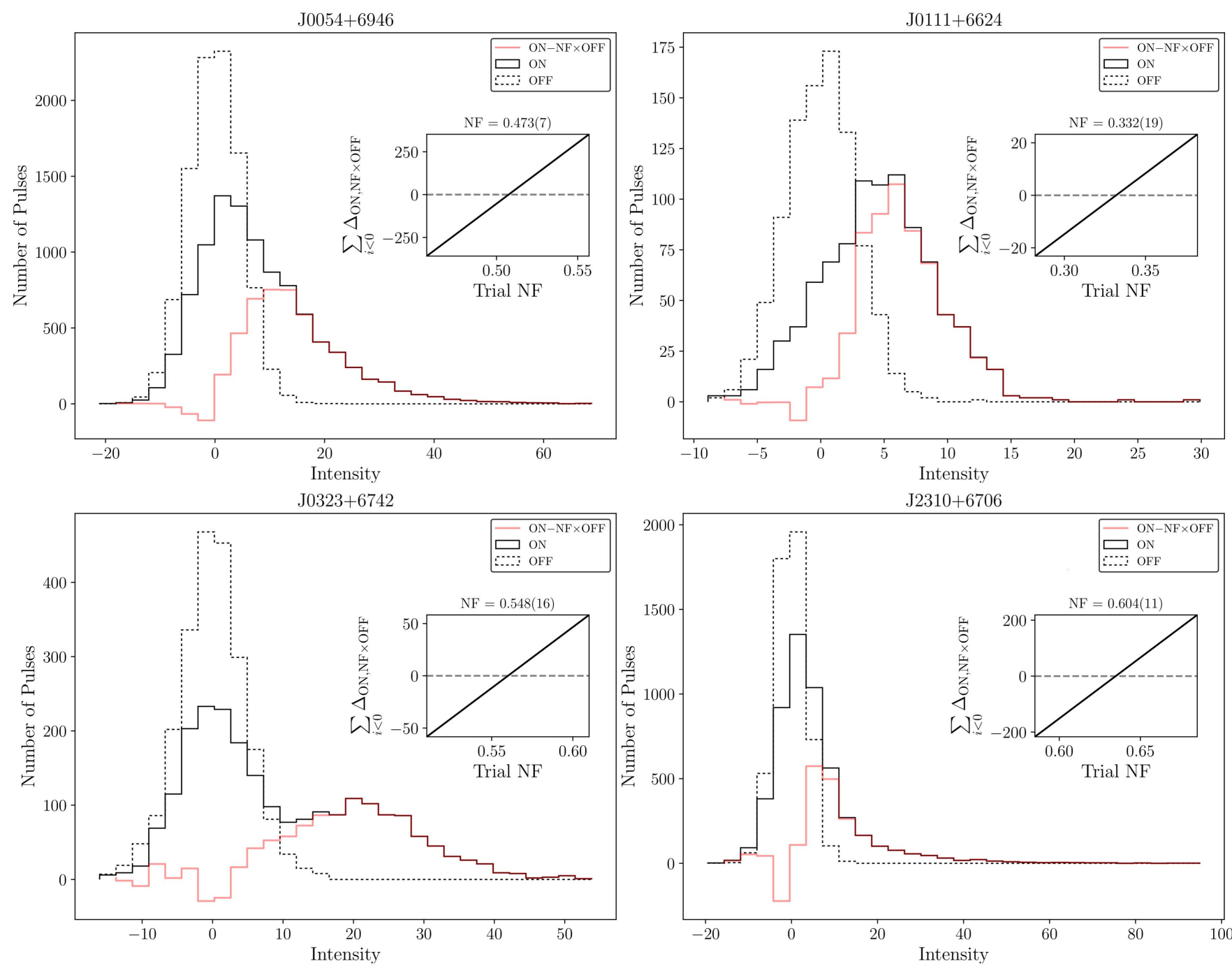

Figure 6. Histograms of summed intensities in ON/OFF windows for PSRs J0054+6946 (top left), J0111+6624 (top right), J0323+6742 (bottom left), and J2310 +6706 (bottom right) shown with solid/dashed lines, respectively. Inset in each panel are plots showing the difference, ON-NF $\times$ OFF (summed over bins with intensity $<0$ ) vs. trial NF values. The red line shows $\mathrm{ON}-\mathrm{NF} \times \mathrm{OFF}$ for the best NF value (where the summed difference is closest to zero).

spin period, characteristic age, and profile morphology (e.g., Ritchings 1976; Rankin 1986; Biggs 1992; Vivekanand 1995; Wang et al. 2007).

To conduct an initial census of nulling pulsars among the discoveries described in this paper, we visually inspected each pulsar's discovery plot and took note of sources that exhibited obvious intensity variations as a function of time, resulting in 19 nulling candidates. We followed a procedure similar to that described in Ritchings (1976) to investigate which (if any) of these pulsars exhibited measurable nulling behavior. Here we describe the procedure used to process and analyze data for each candidate in order to identify nulling pulsars in this sample.

Once this initial selection was made, we used timing data (at both 350 and $820 \mathrm{MHz}$ ) on all of the candidate nullers to confirm or reject them as nulling pulsars and measure NFs where appropriate. Subbanded timing data were dedispersed and folded modulo the pulsar's spin period, resulting in files containing subintegrations equivalent in length to the pulsar's spin period and 64 frequency channels across the band. The RFI was removed interactively with pazi, part of the PSRCHIVE pulsar-processing software package ${ }^{32}$ (Hotan et al. 2004). After downsampling all cleaned detections in time and frequency, the resulting folded profile was used to determine ON/OFF pulse windows of equal size. In most cases, ON/OFF windows spanned $\lesssim 15$ bins, or about $5 \%$ of a full rotation (256 bins). The ON window was centered on the ON pulse region, and the OFF window was fixed 100 bins away, sampling baseline noise.

Cleaned data were downsampled in frequency, and the remaining bins outside both $\mathrm{ON} / \mathrm{OFF}$ windows were used to subtract a DC offset (mean value) from single pulses; any remaining low-frequency noise was also removed during this stage by fitting out a sixth-order polynomial from the baseline. Examples of resulting single-pulse intensities (and folded profiles) are shown in Figure 5. Summed intensities were recorded for each single pulse in both the ON and OFF windows and then binned over intensity to create histograms (see Figures 6 and 7) for each window. In order to identify pulsars exhibiting some form of nulling behavior, we looked

\footnotetext{
32 http://psrchive.sourceforge.net/
} 

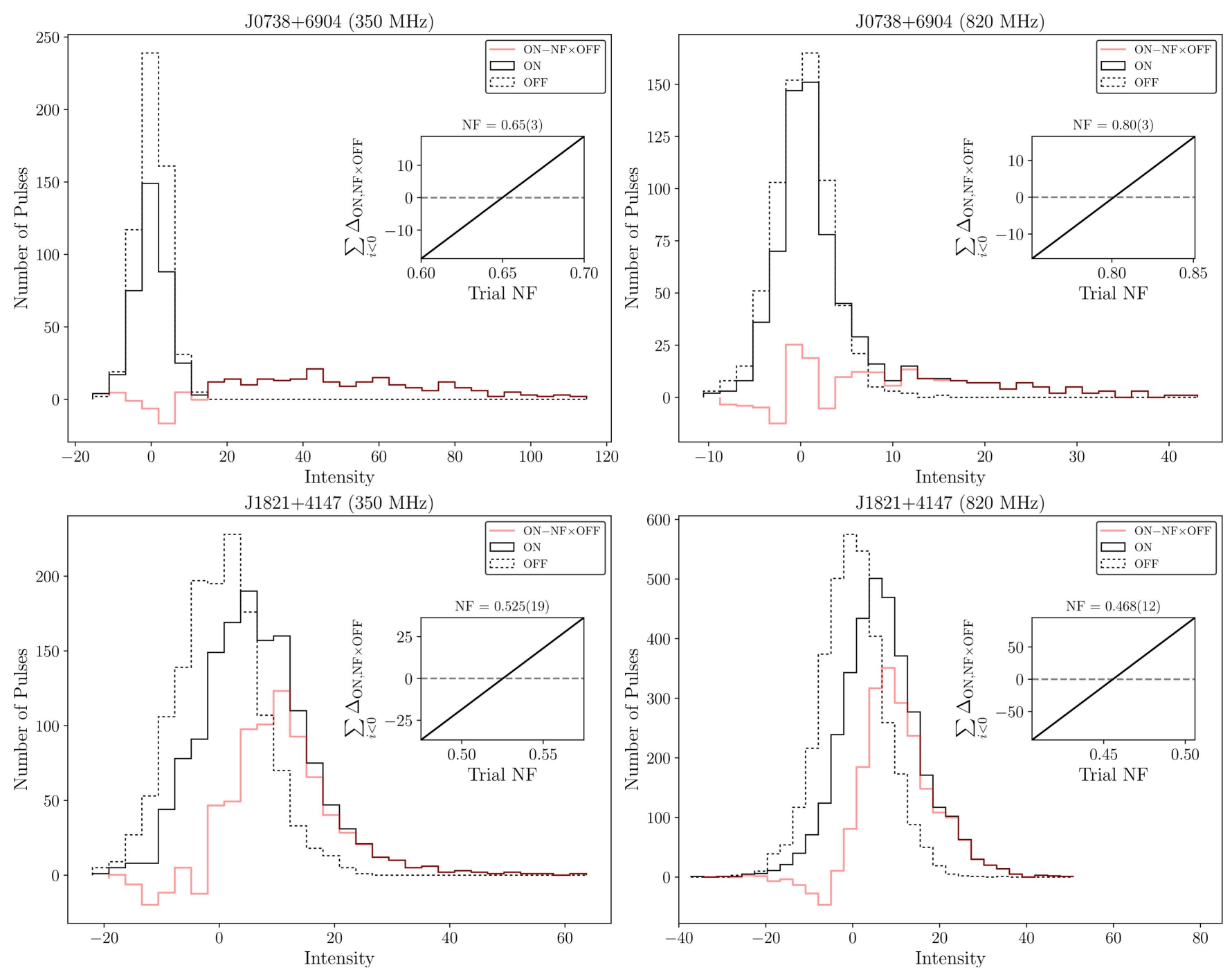

Figure 7. Histograms of summed intensities in ON/OFF windows for PSRs J0738+6904 (350 MHz; top left), J0738+6904 (820 MHz; top right), J1821+4147 (350 MHz; bottom left), and J1821+4147 (820 MHz; bottom right) shown with solid/dashed lines, respectively. Inset in each panel are plots showing the difference, $\mathrm{ON}-\mathrm{NF} \times \mathrm{OFF}$ (summed over bins with intensity $<0$ ) vs. trial NF values. The red line shows $\mathrm{ON}-\mathrm{NF} \times \mathrm{OFF}$ for the best $\mathrm{NF}$ value (where the summed difference is closest to zero).

for sources with ON histograms that showed both measurable single-pulse emission (a tail or positive distribution of $\mathrm{ON}$ intensities) and a distribution of null pulses centered on zero intensity inside the envelope of the OFF histogram (see panel for PSR J0323+6742 in Figure 6 for a canonical example).

Of the 19 candidates for which we carried out this analysis, eight pulsars (group A; PSRs J0612+3721, J1859+7654, $\mathrm{J} 1941+4320, \quad \mathrm{~J} 1954+4357, \quad \mathrm{~J} 2137+6428, \quad \mathrm{~J} 2228+6447$, $\mathrm{J} 2312+6931$, and $\mathrm{J} 2351+8533$ ) were too weak to see single pulses; therefore, the results were inconclusive. Five others (group B; PSRs J0137+6349, J0335+6623, J0944+4106, $\mathrm{J} 1059+6459$, and $\mathrm{J} 1647+6609)$ showed obvious single-pulse emission, but no null distribution was apparent in any of their ON histograms using existing data. Based on our analysis, pulsars from group A have single-pulse emission below our detection threshold, but because of the intensity variability apparent in their discovery plots, we may be able to carry out a similar analysis by summing groups of single pulses (as in Wang et al. 2007) and looking for longer nulls. Pulsars from group B may have extremely low NFs, which may be detectable with extended data sets.
The remaining six pulsars from the original candidate list (PSRs J0054+6946, J0111+6624, J0323+6742, J0738+6904, $\mathrm{J} 1821+4147$, and $\mathrm{J} 2310+6706)$ were identified as new nulling pulsars (see Figure 5 for profiles and examples of their singlepulse behavior). Using their ON/OFF histograms (see Figures 6 and 7), we computed preliminary NFs and found values between 0.33 and 0.80 . For PSRs J0738+6904 and $\mathrm{J} 1821+4147$, timing data from multiple frequencies (350 and $820 \mathrm{MHz}$ ) allowed us to compare nulling behavior across frequencies; based on preliminary results shown in Figure 7, we found similar ON distributions and NF values independent of observing frequency, as also found by Gajjar et al. (2014) on three nulling pulsars.

An analysis that will select nulling pulsars using all existing timing data with an improved method that uses Gaussian mixture models and accounts for the effects of scintillation is under way. Also of interest in a future study would be a detailed comparison between these new sources and the rest of the known nulling population, as well as predictions about the underlying nulling population, given the relatively complete and unbiased nature of the GBNCC survey. 

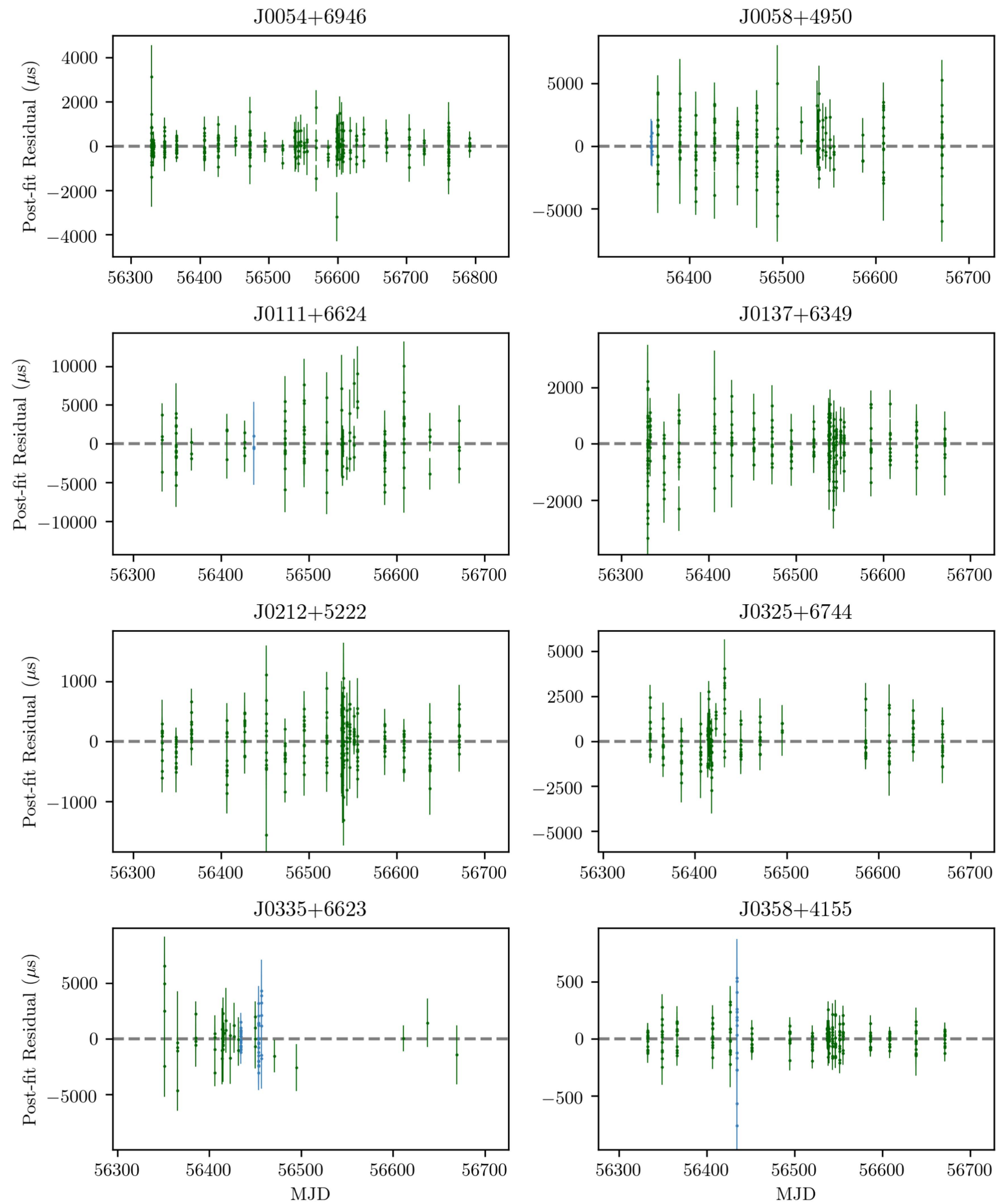

Figure 8. Post-fit timing residuals for the 45 pulsars presented here. Colors correspond to different observing bands: 148 (blue), 350 (orange), 820 (green), 1500 (pink), and $2000 \mathrm{MHz}$ (brown). Note that vertical and horizontal scales differ for each pulsar. (An extended version of this figure is available.) 
J0054+6946

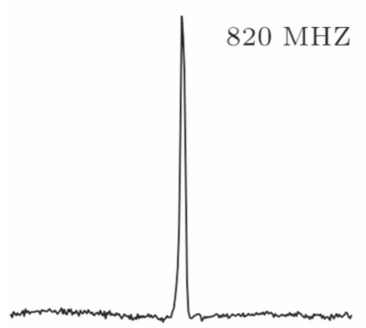

J0212+5222

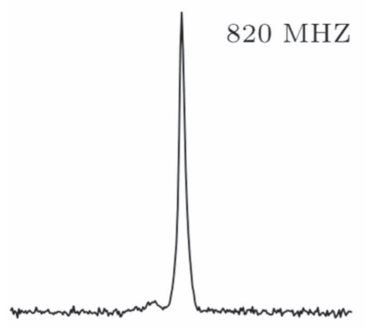

J0358+6627

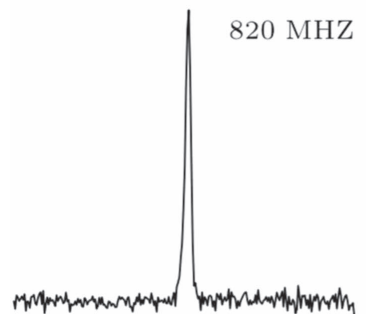

J0738+6904
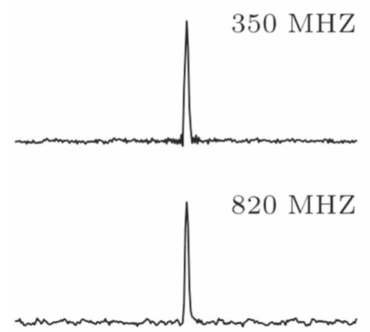

$\mathrm{J} 1059+6459$
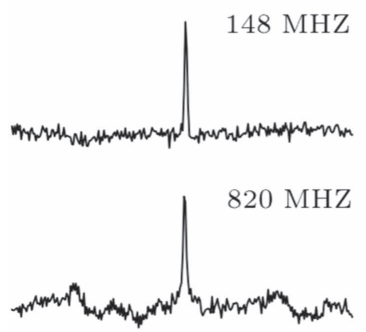

$\mathrm{J} 0058+4950$
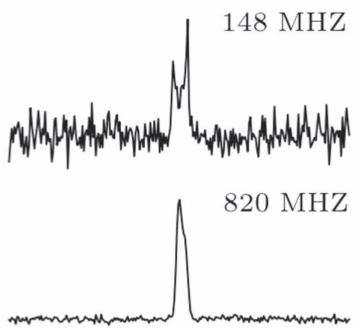

$\mathrm{J} 0325+6744$

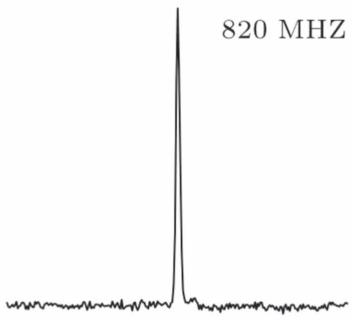

$\mathrm{J} 0509+3801$
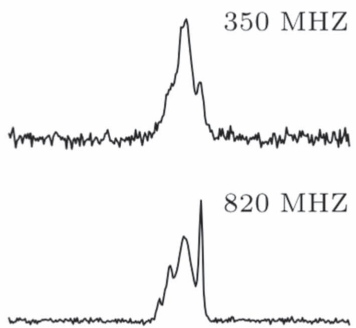

$\mathrm{J} 0740+6620$

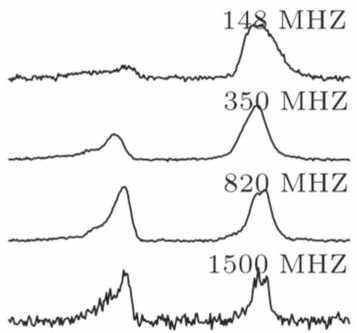

$\mathrm{J} 1125+7819$

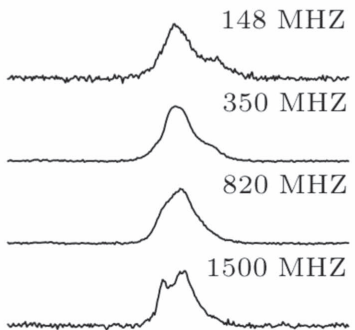

$\mathrm{J} 0111+6624$

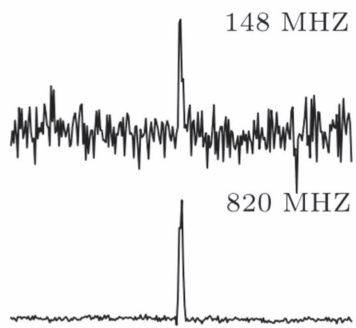

$\mathrm{J} 0335+6623$

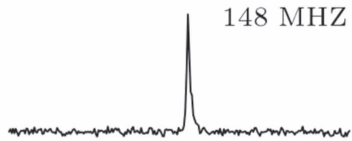

$820 \mathrm{MHZ}$

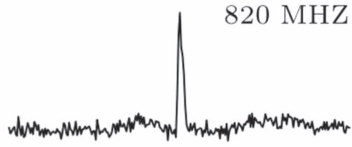

$\mathrm{J} 0518+5416$
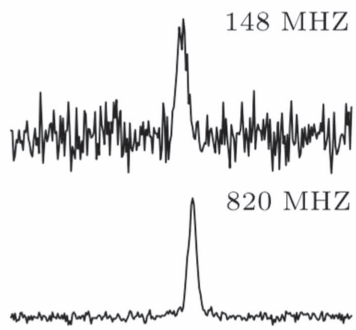

$\mathrm{J} 0747+6646$
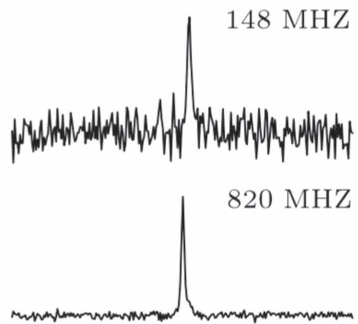

$\mathrm{J} 1624+8643$

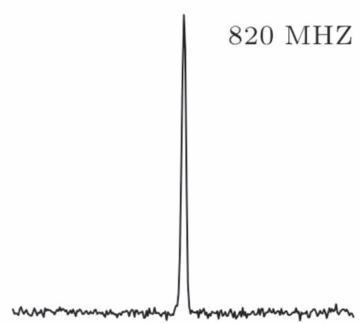

$\mathrm{J} 0137+6349$

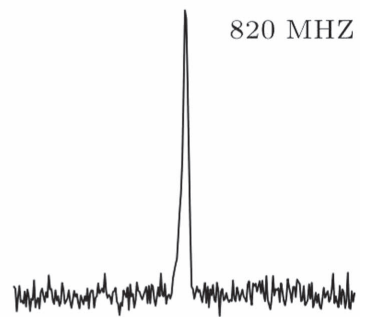

J0358+4155

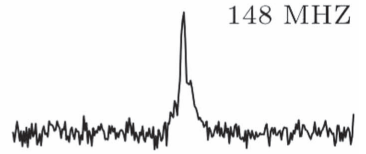

$820 \mathrm{MHZ}$

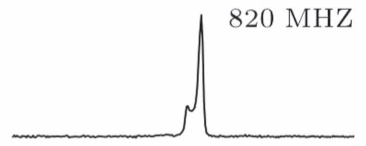

$\mathrm{J} 0612+3721$
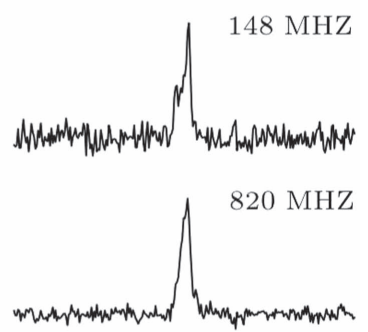

J0944+4106

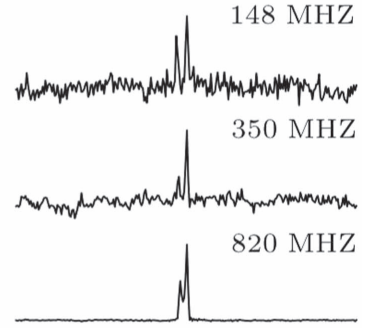

$\mathrm{J} 1628+4406$

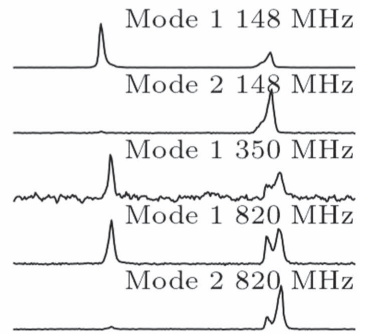

Figure 9. Average pulse profiles for the 45 pulsars presented here. Data from all available observing bands are presented. All profiles have been rotated $180^{\circ}$ from the zero-point pulse phase reference. 


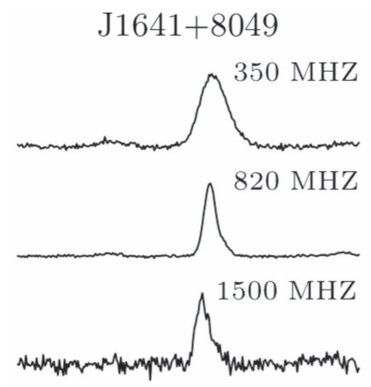

$\mathrm{J} 1815+5546$

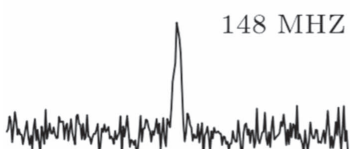

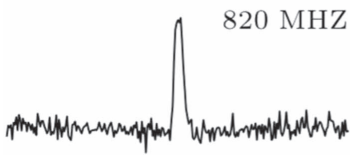

J1934+5219

| $820 \mathrm{MHZ}$

J1954+4357

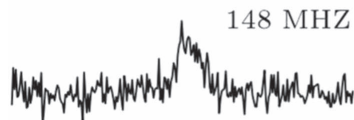

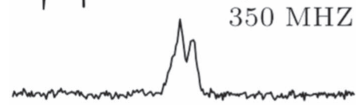

$820 \mathrm{MHZ}$ 1

$\mathrm{J} 2027+7502$
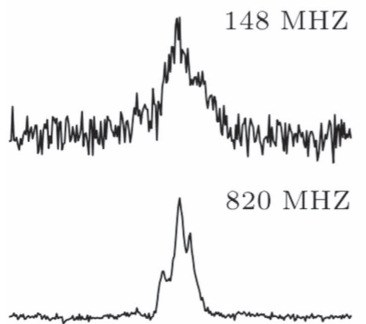

$\mathrm{J} 1647+6608$

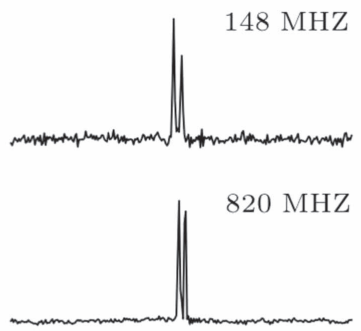

$\mathrm{J} 1821+4147$

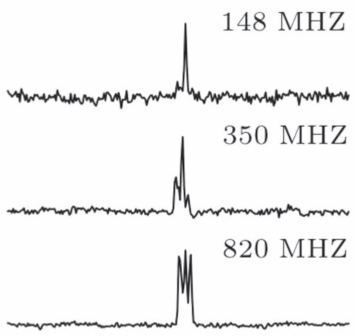

$\mathrm{J} 1938+6604$

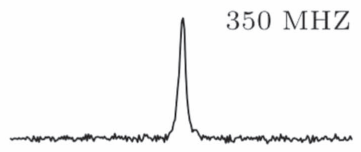

$820 \mathrm{MHZ}$

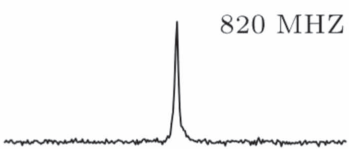

$\mathrm{J} 1955+6708$

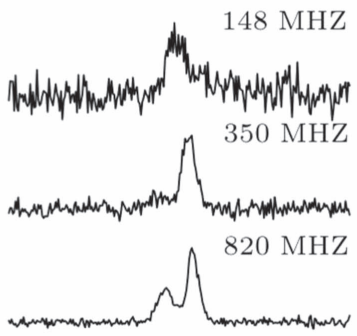

$\mathrm{J} 2123+5434$

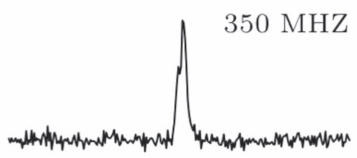

$820 \mathrm{MHZ}$
$\mathrm{J} 1710+4923$

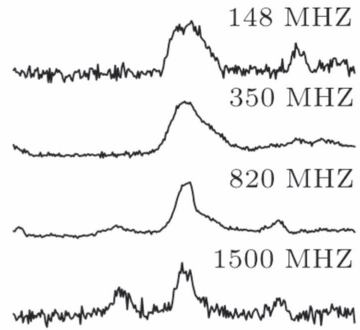

$\mathrm{J} 1859+7654$

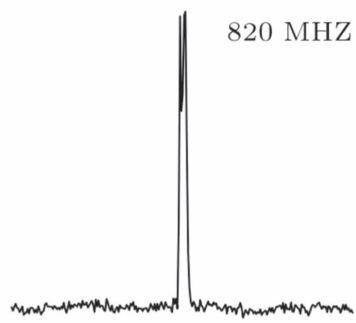

$\mathrm{J} 1941+4320$

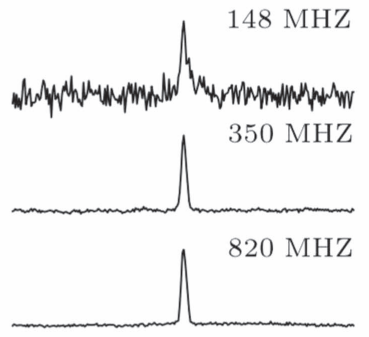

$\mathrm{J} 2001+4258$

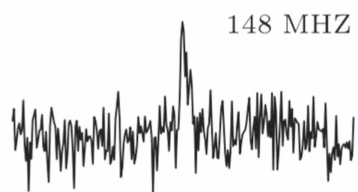

$820 \mathrm{MHZ}$

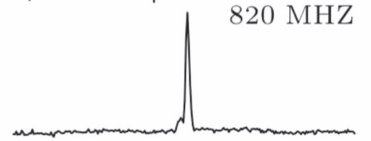

$\mathrm{J} 2137+6428$
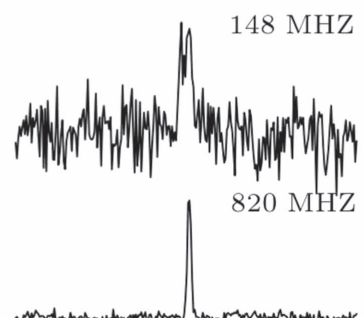

$\mathrm{J} 1800+5034$

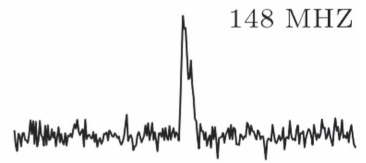

$820 \mathrm{MHZ}$

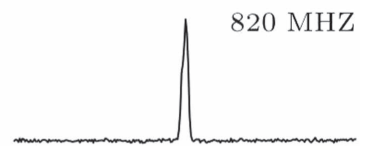

$\mathrm{J} 1923+4243$
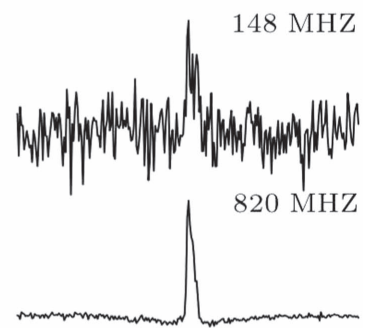

J1942+8106

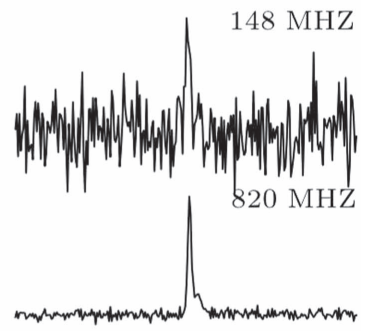

J2017+5906
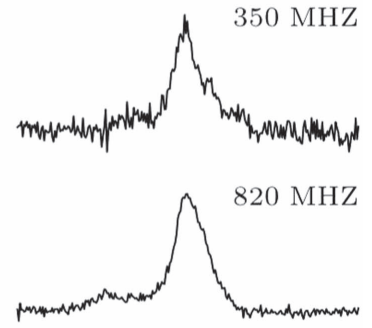

$\mathrm{J} 2208+4056$

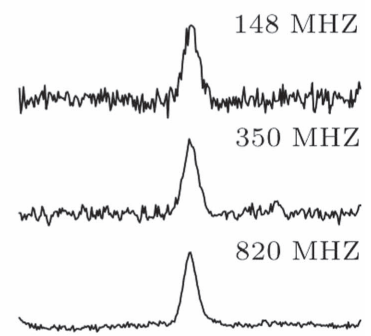

Figure 9. (Continued.) 


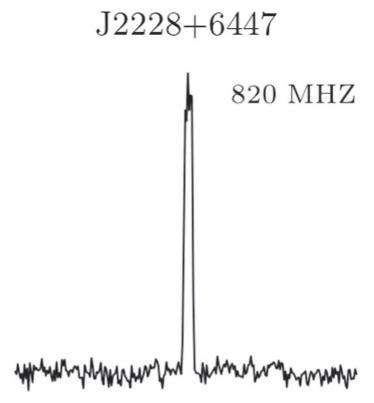

$\mathrm{J} 2241+6941$
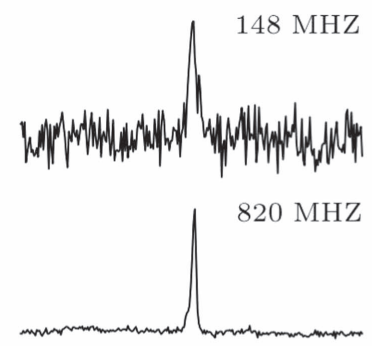

$\mathrm{J} 2310+6706$

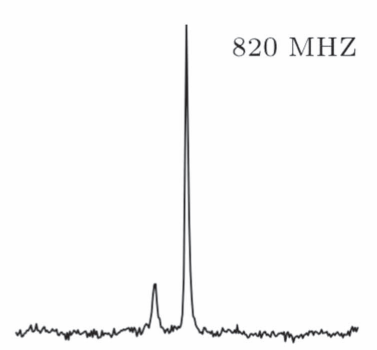

$\mathrm{J} 2312+6931$

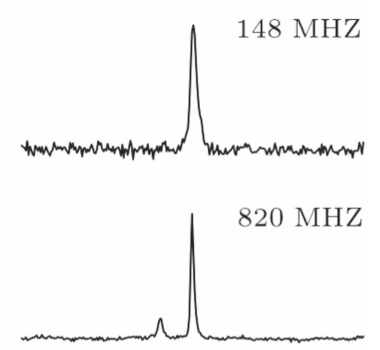

$\mathrm{J} 2351+8533$
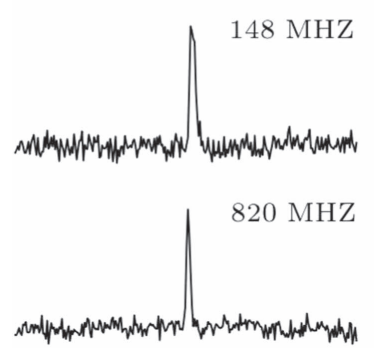

Figure 9. (Continued.)

Table 8

Optical Counterpart Limits

\begin{tabular}{|c|c|c|c|c|c|}
\hline Source & $\begin{array}{c}\text { Companion } \text { Mass }^{\mathrm{a}} \\
\left(M_{\odot}\right)\end{array}$ & Companion Type & $\begin{array}{l}T_{\text {eff }} \\
(\mathrm{K})\end{array}$ & $\begin{array}{l}\text { Constraining Filter } \\
(g / r / i / z)\end{array}$ & $\begin{array}{l}\text { WD Age } \\
\text { (Gyr) }\end{array}$ \\
\hline \multirow[t]{2}{*}{$\mathrm{J} 0740+6620$} & 0.20 & $\mathrm{He}$ WD & $<4200$ & $\bar{i}$ & $>3.2$ \\
\hline & 0.23 & & $<4400$ & & $>4.0$ \\
\hline \multirow[t]{2}{*}{$\mathrm{J} 1125+7819$} & 0.29 & $\mathrm{He} W D$ & $<4400$ & $i$ & $>3.3$ \\
\hline & 0.33 & & $<3500$ & & $>5.6$ \\
\hline \multirow[t]{2}{*}{ J1938+6604 } & 0.87 & CO WD & $<20000$ & $g$ & $>0.179$ \\
\hline & 1.04 & & $<25000$ & & $>0.118$ \\
\hline
\end{tabular}

Note. We use the larger of the DM-inferred distance from either the NE2001 or YMW16 models in setting limits.

${ }^{\mathrm{a}}$ Minimum and median companion masses displayed for each source, assuming $m_{\mathrm{p}}=1.35 M_{\odot}$ and orbital inclination angles of $90^{\circ}$ and $60^{\circ}$, respectively.

\section{Optical Constraints}

Each of the MSPs with a binary companion and nearly circular orbit (see Table 5) most likely has a WD companion. For all of them, we looked for optical counterparts using data from the PanSTARRS $3 \pi$ Steradian Survey (Chambers et al. 2016). By manually inspecting individual (grizy) bands from the PanSTARRS survey's PS1 data release, we found a counterpart for PSR J1641+8049 (Section 5.3.5), but none were found for the three other sources. For nondetections, we used the minimum/median companion masses derived from the systems' binary parameters (see Table 8) and the average $5 \sigma$ magnitude lower limits for the PS1 grizy bands (23.3, 23.2, 23.1, 22.3, and 21.4, respectively; Chambers et al. 2016) to place constraints on the properties of each MSP's WD companion. Based on computed minimum companion masses, PSRs $\mathrm{J} 0740+6620$ and $\mathrm{J} 1125+7819$ likely have He WD companions, and PSR J1938+6604 likely has a CO WD companion.

Reddening was estimated using a 3D map of interstellar dust reddening by Green et al. (2015), the pulsar's sky position, and $D_{\text {DM }}$ (see Tables 4 and 5; we used the larger of the DMinferred distances from the NE2001 or YMW16 models to calculate the most conservative limits). These values were converted to extinctions in each PS1 band using Table 6 from Schlafly \& Finkbeiner (2011). The resulting magnitude limits were translated to upper limits on WD effective temperatures ( $\left.T_{\text {eff }}\right)$ using models for WD mass, radius, and temperature from Istrate et al. (2016). The best WD $T_{\text {eff }}$ constraints are listed for each pulsar's minimum/median companion mass in Table 8 , along with the PS1 bands providing those constraints, and modeled WD cooling ages (Istrate et al. 2016). Because of its significantly higher companion mass, we used different models $^{33}$ (Bergeron et al. 2011; Tremblay et al. 2011) to place constraints on PSR J1938+6604's CO WD but otherwise followed the same procedure described here.

Based on mass and temperature constraints, both PSRs $\mathrm{J} 0740+6620$ and $\mathrm{J} 1125+7819$ have cool He WD companions; for these systems, a correlation between $P_{\mathrm{b}}$ and WD mass is expected (Savonije 1987; Tauris \& Savonije 1999), and the $\left(P_{\mathrm{b}}, m_{\mathrm{WD}}\right)$ relationship predicts companion masses of 0.25 and $0.28 M_{\odot}$, respectively. These are consistent with the minimum companion masses for these systems.

\footnotetext{
33 http://www.astro.umontreal.ca/ bergeron/CoolingModels/
} 


\section{Conclusion}

We present here complete timing solutions for 45 new pulsars discovered by the GBNCC survey. The highlights include two new PTA pulsars that are being used in an effort to detect low-frequency GWs, several intermittent pulsars, a mode-changing pulsar, and five binary pulsars. Among the binary pulsars are a new DNS system, IMBP, and black widow.

These results demonstrate the importance of long-term pulsar timing, as many properties, such as spin-down and proper motion, can only be measured with data spanning 1 yr or more. We continue to observe select sources to improve the measurements of proper motion and some binary parameters. We are also observing approximately 100 additional pulsars discovered in the GBNCC survey, which will be presented in future work. Given our current estimate of survey yield, we expect to discover an additional few dozen long-period pulsars and of order 5-10 MSPs before the survey is completed.

We thank the anonymous referee whose comments improved the quality of this work. The Green Bank Observatory is a facility of the National Science Foundation (NSF) operated under cooperative agreement by Associated Universities, Inc. This paper is based in part on data obtained with the International LOFAR Telescope (ILT). LOFAR (van Haarlem et al. 2013) is the Low Frequency Array designed and constructed by ASTRON. It has facilities in several countries that are owned by various parties (each with their own funding sources) and collectively operated by the ILT foundation under a joint scientific policy. These results also made use of Lowell Observatory's Discovery Channel Telescope. Lowell operates the DCT in partnership with Boston University, Northern Arizona University, the University of Maryland, and the University of Toledo. Partial support of the DCT was provided by Discovery Communications. LMI was built by Lowell Observatory using funds from the NSF (AST-1005313). This paper includes data taken at the McDonald Observatory of the University of Texas at Austin. IRAF is distributed by the National Optical Astronomy Observatory, which is operated by the Association of Universities for Research in Astronomy (AURA) under cooperative agreement with the NSF. J.K.S., M.E.D., F.A.J., D.L.K., M.A.M., S.M.R., K.S., and X.S. are supported by NANOGrav NSF Physics Frontiers Center award number 1430284. MED also acknowledges support from NSF award number AST-1312822. V.I.K. and J.W.T.H. acknowledge funding from an NWO Vidi fellowship and the European Research Council under the European Union's Seventh Framework Programme (FP/2007-2013)/ERC Starting Grant agreement no. 337062 ("DRAGNET"). V.M.K. receives support from an NSERC Discovery Grant and Accelerator Supplement, the Centre de Recherche en Astrophysique du Québec, an R. Howard Webster Foundation Fellowship from the Canadian Institute for Advanced Research, the Canada Research Chairs Program, and the Lorne Trottier Chair in Astrophysics and Cosmology. M.A.M. and B.C. were supported by NSF awards AST-1327526 and OIA-1458952. S.M.R. is a Canadian Institute for Advanced Research Senior Fellow. I.H.S. and pulsar research at UBC are supported by an NSERC Discovery Grant and the Canadian Institute for Advanced Research. J.vL. receives funding from the European Research Council under the European Union's Seventh Framework Programme (FP/2007-2013)/ERC Grant Agreement no. 617199.
Facilities: GBT (GUPPI), LOFAR, Fermi (LAT), DCT (LMI), McDonald Observatory $1 \mathrm{~m}$ (Sinistro).

Software: PRESTO (https://github.com/scottransom/presto), PSRCHIVE (Hotan et al. 2004), TEMPO (http://tempo. sourceforge.net/), PINT (https://github.com/nanograv/PINT), IRAF (Tody 1986, 1993), SCAMP (Bertin \& Arnouts 1996).

\section{ORCID iDs}

Ryan S. Lynch (1D https://orcid.org/0000-0001-5229-7430 Joseph K. Swiggum (ib https://orcid.org/0000-00021075-3837

Vlad I. Kondratiev (i) https://orcid.org/0000-0001-8864-7471

David L. Kaplan (10 https://orcid.org/0000-0001-6295-2881

Kevin Stovall (iD https://orcid.org/0000-0002-7261-594X

Emmanuel Fonseca (i) https://orcid.org/0000-0001-8384-5049

Mallory S. E. Roberts (i) https://orcid.org/0000-00029396-9720

Lina Levin (ib https://orcid.org/0000-0002-2034-2986

Megan E. DeCesar (i) https://orcid.org/0000-0002-2185-1790

Bingyi Cui (iD https://orcid.org/0000-0003-3222-1302

S. Bradley Cenko (i) https://orcid.org/0000-0003-1673-970X

Pradip Gatkine (1D https://orcid.org/0000-0002-1955-2230

Anne M. Archibald (1) https://orcid.org/0000-0003-0638-3340

Pragya Chawla (i) https://orcid.org/0000-0002-3426-7606

Jason W. T. Hessels (iD https://orcid.org/0000-0003-

2317-1446

Victoria M. Kaspi (10 https://orcid.org/0000-0001-9345-0307

Maura A. McLaughlin (1) https://orcid.org/0000-00017697-7422

Hind Al Noori (D) https://orcid.org/0000-0002-4187-4981

Scott M. Ransom (iD https://orcid.org/0000-0001-5799-9714

Renée Spiewak (1) https://orcid.org/0000-0002-6730-3298

Ingrid H. Stairs (iD https://orcid.org/0000-0001-9784-8670

Joeri van Leeuwen (D) https://orcid.org/0000-0001-8503-6958

\section{References}

Ahn, C. P., Alexandroff, R., Allende Prieto, C., et al. 2014, ApJS, 211, 17 Antoniadis, J., Freire, P. C. C., Wex, N., et al. 2013, Sci, 340, 448 Antoniadis, J., Tauris, T. M., Ozel, F., et al. 2016, arXiv:1605.01665 Arzoumanian, Z., Brazier, A., Burke-Spolaor, S., et al. 2018, ApJS, 235, 37 Backer, D. C. 1970, Natur, 228, 42

Bassa, C. G., Janssen, G. H., Stappers, B. W., et al. 2016, MNRAS, 460, 2207 Bergeron, P., Wesemael, F., Dufour, P., et al. 2011, ApJ, 737, 28

Bertin, E. 2006, in ASP Conf. Ser 351, Astronomical Data Analysis Software and Systems XV, ed. C. Gabriel et al., 112

Bertin, E., \& Arnouts, S. 1996, A\&AS, 117, 393

Bhattacharyya, B., \& Nityananda, R. 2008, MNRAS, 387, 273

Biggs, J. D. 1992, ApJ, 394, 574

Camilo, F., Kerr, M., Ray, P. S., et al. 2015, ApJ, 810, 85

Camilo, F., Lyne, A. G., Manchester, R. N., et al. 2001, ApJL, 548, L187

Capitaine, N., Wallace, P. T., \& Chapront, J. 2003, A\&A, 412, 567

Chambers, K. C., Magnier, E. A., Metcalfe, N., et al. 2016, arXiv:1612.05560

Chawla, P., Kaspi, V. M., Josephy, A., et al. 2017, arXiv:1701.07457

Cordes, J. M., \& Lazio, T. J. W. 2002, arXiv:0207156

de Jager, O. C., Raubenheimer, B. C., \& Swanepoel, J. W. H. 1989, A\&A, 221,180

Demorest, P. B., Pennucci, T., Ransom, S. M., Roberts, M. S. E., \& Hessels, J. W. T. 2010, Natur, 467, 1081

Espinoza, C. M., Guillemot, L., Çelik, Ö, et al. 2013, MNRAS, 430, 571 Ferdman, R. D., Stairs, I. H., Kramer, M., et al. 2013, ApJ, 767, 85

Fruchter, A. S., Stinebring, D. R., \& Taylor, J. H. 1988, Natur, 333, 237 Gajjar, V. 2017, arXiv:1706.05407

Gajjar, V., Joshi, B. C., Kramer, M., Karuppusamy, R., \& Smits, R. 2014, ApJ, 797, 18

Gonzalez, M. E., Stairs, I. H., Ferdman, R. D., et al. 2011, ApJ, 743, 102 Green, G. M., Schlafly, E. F., Finkbeiner, D. P., et al. 2015, ApJ, 810, 25 
Hobbs, G. 2013, CQGra, 30, 224007

Hobbs, G., Lorimer, D. R., Lyne, A. G., \& Kramer, M. 2005, MNRAS, 360,974

Hotan, A. W., van Straten, W., \& Manchester, R. N. 2004, PASA, 21, 302

Istrate, A. G., Marchant, P., Tauris, T. M., et al. 2016, A\&A, 595, A35

Kaplan, D. L., Kupfer, T., Nice, D. J., et al. 2016, ApJ, 826, 86

Karako-Argaman, C., Kaspi, V. M., Lynch, R. S., et al. 2015, ApJ, 809, 67

Kawash, A. M., McLaughlin, M. A., Kaplan, D. L., et al. 2018, ApJ, 857, 131

Kiziltan, B., Kottas, A., De Yoreo, M., \& Thorsett, S. E. 2013, ApJ, 778, 66

Kondratiev, V. I., Verbiest, J. P. W., Hessels, J. W. T., et al. 2016, A\&A, 585, A128

Kramer, M., \& Champion, D. J. 2013, CQGra, 30, 224009

Kramer, M., Stairs, I. H., Manchester, R. N., et al. 2006, Sci, 314, 97

Lange, C., Camilo, F., Wex, N., et al. 2001, MNRAS, 326, 274

Lattimer, J. M., \& Prakash, M. 2004, Sci, 304, 536

Lyne, A., Hobbs, G., Kramer, M., Stairs, I., \& Stappers, B. 2010, Sci, 329, 408

Manchester, R. N. \& IPTA 2013, CQGra, 30, 224010

Manchester, R. N., Hobbs, G. B., Teoh, A., \& Hobbs, M. 2005, AJ, 129, 1993

Matthews, A. M., Nice, D. J., Fonseca, E., et al. 2016, ApJ, 818, 92

McLaughlin, M. A. 2013, CQGra, 30, 224008

McLaughlin, M. A., Lyne, A. G., Lorimer, D. R., et al. 2006, Natur, 439, 817

Morris, D. J., Hobbs, G., Lyne, A. G., et al. 2002, MNRAS, 335, 275

Nice, D. J., \& Taylor, J. H. 1995, ApJ, 441, 429

Özel, F., Psaltis, D., Narayan, R., \& Santos Villarreal, A. 2012, ApJ, 757, 55

Phinney, E. S., Evans, C. R., Blandford, R. D., \& Kulkarni, S. R. 1988, Natur, 333,832

Rankin, J. M. 1986, ApJ, 301, 901
Ransom, S. M., Eikenberry, S. S., \& Middleditch, J. 2002, AJ, 124, 1788

Ransom, S. M., Ray, P. S., Camilo, F., et al. 2011, ApJL, 727, L16

Ritchings, R. T. 1976, MNRAS, 176, 249

Roberts, M. S. E. 2013, in Proc. IAU Symp. 291, Neutron Stars and Pulsars: Challenges and Opportunities after 80 years, ed. J. van Leeuwen (Cambridge: Cambridge Univ. Press), 127

Savonije, G. J. 1987, Natur, 325, 416

Schlafly, E. F., \& Finkbeiner, D. P. 2011, ApJ, 737, 103

Shklovskii, I. S. 1970, SvA, 13, 562

Siemens, X., Ellis, J., Jenet, F., \& Romano, J. D. 2013, CQGra, 30, 224015

Splaver, E. M., Nice, D. J., Arzoumanian, Z., et al. 2002, ApJ, 581, 509

Stovall, K., Lynch, R. S., Ransom, S. M., et al. 2014, ApJ, 791, 67

Tauris, T. M., \& Savonije, G. J. 1999, A\&A, 350, 928

Taylor, J. H. 1992, RSPTA, 341, 117

Taylor, J. H., \& Weisberg, J. M. 1989, ApJ, 345, 434

Tody, D. 1986, Proc. SPIE, 627, 733

Tody, D. 1993, in ASP Conf. Ser 52, Astronomical Data Analysis Software and Systems II, ed. R. J. Hanisch, R. J. V. Brissenden, \& J. Barnes (San Francisco, CA: ASP), 173

Toscano, M., Sandhu, J. S., Bailes, M., et al. 1999, MNRAS, 307, 925

Tremblay, P.-E., Bergeron, P., \& Gianninas, A. 2011, ApJ, 730, 128

van Haarlem, M. P., Wise, M. W., Gunst, A. W., et al. 2013, A\&A, 556, A2

Vivekanand, M. 1995, MNRAS, 274, 785

Wang, N., Manchester, R. N., \& Johnston, S. 2007, MNRAS, 377, 1383

Weisberg, J. M., Nice, D. J., \& Taylor, J. H. 2010, ApJ, 722, 1030

Wu, J. H. K., Kong, A. K. H., Huang, R. H. H., et al. 2012, ApJ, 748, 141

Yao, J. M., Manchester, R. N., \& Wang, N. 2017, ApJ, 835, 29

Zhu, W. W., Berndsen, A., Madsen, E. C., et al. 2014, ApJ, 781, 117 